\title{
Epigenome-wide association study of lung function level and its change
}

\author{
Medea Imboden ${ }^{1,2,35}$, Matthias Wielscher ${ }^{3,4,35}$, Faisal I. Rezwan ${ }^{5,35}$ \\ André F.S. Amaral $\mathbb{1}^{3,6,35}$, Emmanuel Schaffner ${ }^{1,2}$, Ayoung Jeong ${ }^{1,2}$, \\ Anna Beckmeyer-Borowko (10) ${ }^{1,2}$, Sarah E. Harris ${ }^{7,8}$, John M. Starr ${ }^{7,9,+}$, \\ Ian J. Deary ${ }^{7,10}$, Claudia Flexeder ${ }^{11}$, Melanie Waldenberger ${ }^{11,12}$, \\ Annette Peters ${ }^{11,12}$, Holger Schulz ${ }^{11,13}$, Su Chen ${ }^{14}$, Shadia Khan Sunny ${ }^{15}$ \\ Wilfried J.J. Karmaus ${ }^{15}$, Yu Jiang ${ }^{15}$, Gertraud Erhart ${ }^{16}$, Florian Kronenberg ${ }^{16}$, \\ Ryan Arathimos ${ }^{17,18}$, Gemma C. Sharp ${ }^{17,18,19}$, Alexander John Henderson ${ }^{18}$, \\ Yu Fu ${ }^{20}$, Päivi Piirilä ${ }^{21}$, Kirsi H. Pietiläinen ${ }^{22,23}$, Miina Ollikainen ${ }^{20}$, \\ Asa Johansson ${ }^{24}$, Ulf Gyllensten ${ }^{24}$, Maaike de Vries (10) ${ }^{25,26}$, \\ Diana A. van der Plaat ${ }^{25,26}$, Kim de Jong ${ }^{25,26}$, H. Marike Boezen ${ }^{25,26}$ \\ lan P. Hall ${ }^{27,28}$, Martin D. Tobin ${ }^{29,30}$, Marjo-Riitta Jarvelin ${ }^{4,31,32,33,34,35}$ \\ John W. Holloway $\mathbb{1}^{5,35}$, Deborah Jarvis ${ }^{3,6,35}$ and Nicole M. Probst-Hensch ${ }^{1,2,35}$
}

@ERSpublications

An agnostic association study on lung function using longitudinal population-based cohort data shows that differentially methylated genomic sites related to smoking are strongly associated with lung function in adults http://ow.ly/wYID30onUB4

Cite this article as: Imboden M, Wielscher M, Rezwan FI, et al. Epigenome-wide association study of lung function level and its change. Eur Respir J 2019; 54: 1900457 [https://doi.org/10.1183/13993003.00457-2019].

ABSTRACT Previous reports link differential DNA methylation (DNAme) to environmental exposures that are associated with lung function. Direct evidence on lung function DNAme is, however, limited. We undertook an agnostic epigenome-wide association study (EWAS) on pre-bronchodilation lung function and its change in adults.

In a discovery-replication EWAS design, DNAme in blood and spirometry were measured twice, 6-15 years apart, in the same participants of three adult population-based discovery cohorts $(n=2043)$. Associated DNAme markers $\left(\mathrm{p}<5 \times 10^{-7}\right)$ were tested in seven replication cohorts (adult: $\mathrm{n}=3327$; childhood: $n=420$ ). Technical bias-adjusted residuals of a regression of the normalised absolute $\beta$-values on control probe-derived principle components were regressed on level and change of forced expiratory volume in $1 \mathrm{~s}$ (FEV1), forced vital capacity (FVC) and their ratio (FEV1/FVC) in the covariate-adjusted discovery EWAS. Inverse-variance-weighted meta-analyses were performed on results from discovery and replication samples in all participants and never-smokers.

EWAS signals were enriched for smoking-related DNAme. We replicated 57 lung function DNAme markers in adult, but not childhood samples, all previously associated with smoking. Markers not previously associated with smoking failed replication. cg05575921 (AHRR (aryl hydrocarbon receptor repressor)) showed the statistically most significant association with cross-sectional lung function (FEV1/FVC: pdiscovery $=3.96 \times 10^{-21}$ and pcombined $=7.22 \times 10^{-50}$ ). A score combining 10 DNAme markers previously reported to mediate the effect of smoking on lung function was associated with lung function (FEV $1 / F V C$ : $p=2.65 \times 10^{-20}$ ).

Our results reveal that lung function-associated methylation signals in adults are predominantly smoking related, and possibly of clinical utility in identifying poor lung function and accelerated decline. Larger studies with more repeat time-points are needed to identify lung function DNAme in never-smokers and in children.

This article has supplementary material available from erj.ersjournals.com

Data available from the Dryad Digital Repository: https://doi.org/10.5061/dryad.pr10c20

Received: Sept 242018 | Accepted after revision: March 272019

Copyright OERS 2019. This version is distributed under the terms of the Creative Commons Attribution Licence 4.0. 


\section{Introduction}

Lung function has an estimated heritability of between $30 \%$ and $70 \%$ [1]. The variance in phenotype remains incompletely explained by genetic variation, but the impact of environmental exposure on respiratory health and lung function over the life course is well recognised. In particular, pro-inflammatory and oxidative inhalants such as cigarette and environmental tobacco smoke, air pollution, and occupational exposures are important contributors to the increased risk of respiratory symptoms, accelerated lung function decline in adults and poor lung growth in children. DNA methylation (DNAme) has been associated with a wide variety of traits and chronic diseases.

A large body of evidence including results from epigenome-wide association studies (EWASs) shows differentially methylated CpG (5'-cytosine-phosphate-guanine-3' dinucleotide) sites throughout the genome in response to environmental exposures, in particular cigarette smoking [2-4]. In contrast, reports of DNAme associated with respiratory diseases and lung function show inconsistent findings [5, 6]. Most recently, however, independent reports pointed to the consistent association of DNAme in the AHRR gene, cg05575921, with lung function in adults $[4,6,7]$.

The current study aimed at agnostically identifying lung function-specific DNAme signals. We undertook a covariate-adjusted EWAS using questionnaire data, spirometry and peripheral blood samples collected in the same participants (discovery cohorts: ECRHS (European Community Respiratory Health Study), NFBC1966 (Northern Finland Birth Cohort 1966) and SAPALDIA (Swiss Study on Air Pollution Heart and Lung Disease in Adults); cohort description in supplementary material) at two time-points 6-15 years apart. EWAS analyses were performed on lung function parameters of forced expiratory volume in $1 \mathrm{~s}$ (FEV1), forced vital capacity (FVC) and their ratio (FEV1/FVC). The analyses focused on cross-sectional associations at different time-points and on identifying DNAme markers predicting change in lung function. We tested discovery-identified CpGs $\left(\mathrm{p}<5 \times 10^{-7}\right.$ for at least one lung function parameter) for replication in adult samples from five adult cohorts (LBC1936 (Lothian Birth Cohort 1936, adult inception birth cohort), KORA (Cooperative Health Research in the Augsburg Region Study), LifeLines (LifeLines cohort study), NSPHS (North Sweden Population Health Study) and FTC (Finnish Twin Cohort study))

Affiliations: ${ }^{1}$ Chronic Disease Epidemiology Unit, Dept of Epidemiology and Public Health, Swiss Tropical and Public Health Institute, Basel, Switzerland. ${ }^{2}$ University of Basel, Basel, Switzerland. ${ }^{3} \mathrm{MRC}$-PHE Centre for Environment and Health, Imperial College London, London, UK. ${ }^{4}$ Dept of Epidemiology and Biostatistics, School of Public Health, Imperial College London, London, UK. ${ }^{5}$ Human Development and Health, Faculty of Medicine, University of Southampton, Southampton, UK. ${ }^{6}$ Population Health and Occupational Disease, NHLI, Imperial College London, London, UK. ${ }^{7}$ Centre for Cognitive Ageing and Cognitive Epidemiology, University of Edinburgh, Edinburgh, UK. ${ }^{8}$ Medical Genetics Section, University of Edinburgh Centre for Genomic and Experimental Medicine and MRC Institute of Genetics and Molecular Medicine, Western General Hospital, Edinburgh, UK. ${ }^{9}$ Alzheimer Scotland Dementia Research Centre, University of Edinburgh, Edinburgh, UK. ${ }^{10}$ Dept of Psychology, University of Edinburgh, Edinburgh, UK. ${ }^{11}$ Institute of Epidemiology, Helmholtz Zentrum München, German Research Center for Environmental Health, Neuherberg, Germany. ${ }^{12}$ Research Unit Molecular Epidemiology, Helmholtz Zentrum München, German Research Center for Environmental Health, Neuherberg, Germany. ${ }^{13}$ Comprehensive Pneumology Center Munich (CPC-M), Member of the German Center for Lung Research (DZL), Munich, Germany. ${ }^{14}$ Dept of Mathematical Sciences, University of Memphis, Memphis, TN, USA. ${ }^{15}$ Division of Epidemiology, Biostatistics, and Environmental Health, School of Public Health, University of Memphis, Memphis, TN, USA. ${ }^{16}$ Division of Genetic Epidemiology, Dept of Medical Genetics, Molecular and Clinical Pharmacology, Medical University of Innsbruck, Innsbruck, Austria. ${ }^{17} \mathrm{MRC}$ Integrative Epidemiology Unit, University of Bristol, Bristol, UK. ${ }^{18}$ Dept of Population Health Sciences, Bristol Medical School, University of Bristol, Bristol, UK. ${ }^{19}$ Bristol Dental School, University of Bristol, Bristol, UK. ${ }^{20}$ Institute for Molecular Medicine Finland (FIMM), University of Helsinki, Helsinki, Finland. ${ }^{21}$ Unit of Clinical Physiology, HUS Medical Imaging Center, Helsinki University Central Hospital and University of Helsinki, Helsinki, Finland. ${ }^{22}$ Obesity Research Unit, Research Programs Unit, University of Helsinki, Helsinki, Finland.

${ }^{23}$ Abdominal Center, Endocrinology, Helsinki University Hospital and University of Helsinki, Helsinki, Finland.

${ }^{24}$ Dept of Immunology, Genetics and Pathology, Science for Life Laboratory, Uppsala University, Uppsala,

Sweden. ${ }^{25}$ University of Groningen, University Medical Center Groningen, Dept of Epidemiology, Groningen, The Netherlands. ${ }^{26}$ Groningen Research Institute for Asthma and COPD (GRIAC), University of Groningen, University Medical Center Groningen, Groningen, The Netherlands. ${ }^{27}$ Division of Respiratory Medicine, University of Nottingham, Nottingham, UK. ${ }^{28}$ National Institute for Health Research, Nottingham Biomedical Research Centre, Nottingham University Hospitals, Nottingham, UK. ${ }^{29}$ Dept of Health Sciences, University of Leicester, Leicester, UK. ${ }^{30}$ National Institute of Health Research Biomedical Research Centre, University of Leicester, Leicester, UK. ${ }^{31}$ Center for Life Course Health Research, Faculty of Medicine, University of Oulu, Oulu, Finland. ${ }^{32}$ Biocenter Oulu, University of Oulu, Oulu, Finland. ${ }^{33}$ Unit of Primary Health Care, Oulu University Hospital, Oulu, Finland. ${ }^{34}$ Dept of Life Sciences, College of Health and Life Sciences, Brunel University London, London, UK. ${ }^{35}$ These authors contributed equally to this work.

Correspondence: Medea Imboden, Chronic Disease Epidemiology Unit, Dept of Epidemiology and Public Health, Swiss Tropical and Public Health Institute, Socinstrasse 57, 4002 Basel, Switzerland.

E-mail: medea.imbodendswisstph.ch 
and in childhood samples from two birth cohorts (ALSPAC (Avon Longitudinal Study of Parents and Children) and IOWBC (Isle of Wight Birth Cohort)).

\section{Methods}

\section{Study design and participants}

The discovery sample ( $\mathrm{n}=2043$ ) comprised three population-based cohort studies, part of the Aging Lungs in European Cohorts (ALEC) project. ECRHS $(n=470)$ and SAPALDIA $(n=962)$ are adult cohorts designed to investigate respiratory health. NFBC1966 $(n=611)$ is a birth cohort with follow-up to adult age. The replication sample consisted of five adult cohorts (KORA $(n=628)$, LifeLines $(n=1622)$, NSPHS $(\mathrm{n}=535)$, LBC1936 $(\mathrm{n}=449)$ and FTC $(\mathrm{n}=93))$ and two childhood birth cohorts (ALSPAC $(\mathrm{n}=258)$ and IOWBC $(n=162)$ ). Replication data from two time-points were available only for KORA and LBC1936 (adult) and ALSPAC and IOWBC (childhood). For cohort details and contribution to analysis, refer to the supplementary material and supplementary figure S1.

All cohorts comply with the Declaration of Helsinki, and ethical approval was obtained from the respective national and regional ethical review committees.

\section{Procedures}

In the discovery cohorts, DNAme measurements using Infinium technology (Illumina, San Diego, CA, USA) were obtained from peripheral blood samples collected at two consecutive follow-up surveys several years apart. The Infinium 450K BeadChip was used for samples of 984 SAPALDIA participants from both time-points and of 732 NFBC1966 participants collected at time-point 1. The Infinium EPIC BeadChip was used for samples of 509 ECRHS participants from both time-points and of 716 NFBC1966 participants collected at time-point 2. For cohort-specific EWAS analyses, we used all autosomal markers available for each time-point and cohort-specific EWAS marker results were meta-analysed without restriction to markers common to both arrays. DNAme data used for replication were restricted to discovery-identified (sentinel) CpGs and analysed on various arrays.

Epidemiological data, including covariate information at the subject level, were collected by interviewassisted questionnaires and objective measures. Pre-bronchodilation spirometric data were obtained by performing American Thoracic Society/European Respiratory Society-compliant spirometry (supplementary material).

\section{Statistical analyses}

Epigenome-wide methylation data were analysed in $\mathrm{R}$ version 3.4.3 ( $\mathrm{R}$ Foundation for Statistical Computing, Vienna, Austria). Differential blood cell count was estimated using a reference dataset and the $\mathrm{R}$ package minfi $[8,9]$. DNAme used as predictors in the statistical models for the adult cohorts were obtained by deriving residuals from linear regression of the normalised absolute DNAme ( $\beta$-values) on the Illumina control probe-derived 30 first principal components to correct for correlation structures within the data, including technical bias. Thus, effect sizes reported here of the association are not comparable to effect sizes reported elsewhere using normalised $\beta$-values as predictor. In the childhood data, batch effect was corrected at the analysis level by regressing the DNAme values against the technical covariates.

Epigenome-wide covariate-adjusted linear regression was performed to assess the association of single CpG markers with FEV1 (L), FVC (L), their ratio (FEV1/FVC) and their change during follow-up. This multilevel EWAS design tested different models in all participants and never-smoking participants (figure 1). First, cross-sectional EWASs were examined separately at time-point 1 (EWAS1) and time-point 2 (EWAS2) to assess the consistency of the association over follow-up time. Second, the association of DNAme at the first time-point (DNAme1) with change in lung function during follow-up was assessed (prediction EWAS (EWASpredict)). Covariate-adjusted mixed linear regressions with a random intercept on the subject were undertaken using data from both time-points (repeat cross-sectional analysis (EWASrepeat)).

All associations were adjusted for a set of a priori selected covariates known to influence respiratory outcomes from previous research conducted by SAPALDIA and ECRHS. The covariate-adjusted model (Mbase) included age, age squared, height, squared deviation from the mean of height, sex and interaction terms of sex with four covariates (age, age squared, height and squared deviation of height), education, body mass index, spirometer type, study centre as well as estimated cell composition (CD8 cells, CD4 cells, natural killer cells, B-cells, monocytes, eosinophils and neutrophils). Analyses in all participants were run without (Mbase) and with additional smoking adjustment including smoking status and pack-years (Msmok). Mbase covariate adjustment was applied in never-smokers. Prediction associations of DNAme1 were additionally adjusted for lung function at time-point 1 . The same covariate adjustment was applied in adult replication analyses, whereas childhood covariates did not include squared terms. 


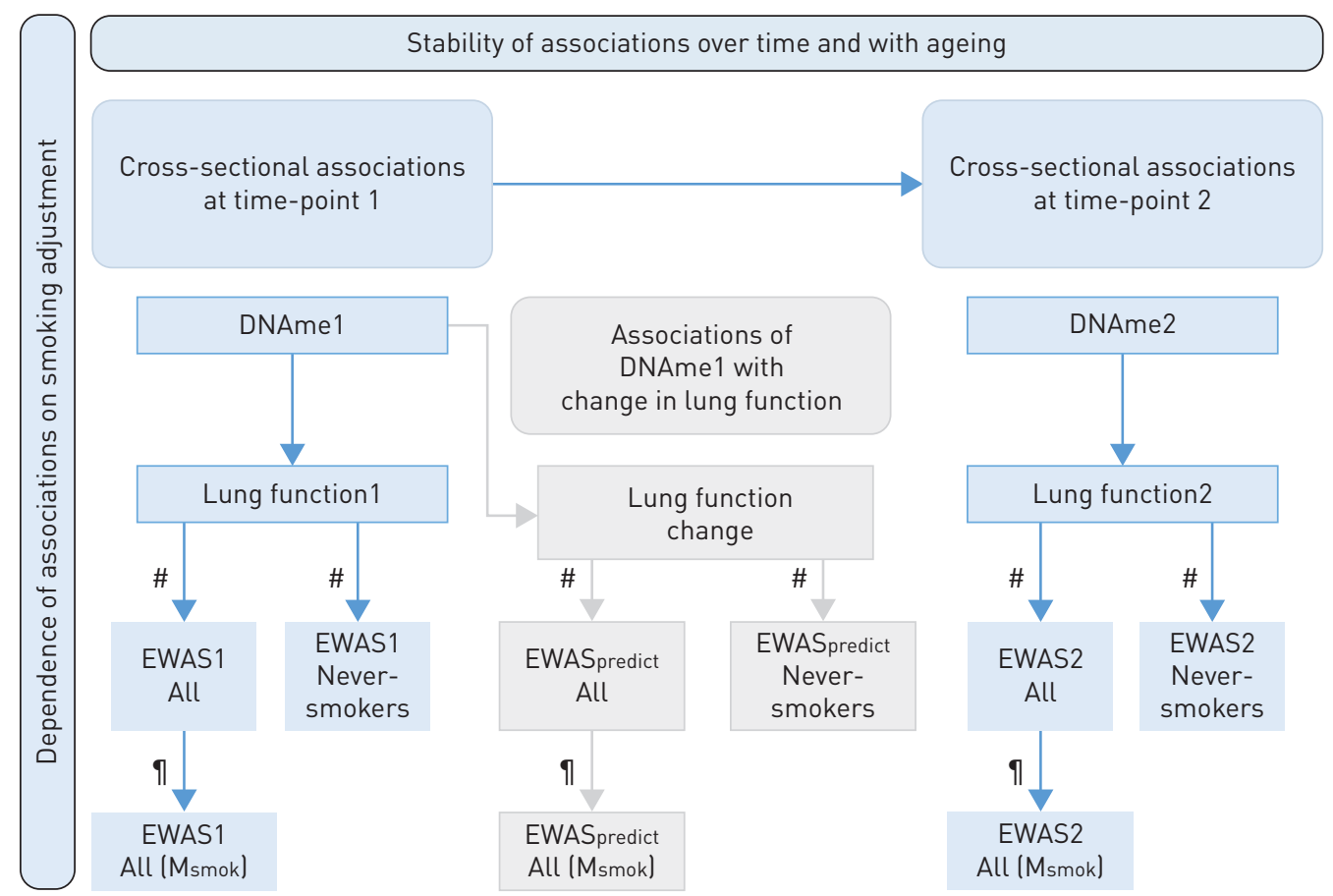

FIGURE 1 Flow of the multilevel discovery design of the epigenome-wide association study (EWAS) on lung function parameters: forced expiratory volume in $1 \mathrm{~s}$ (FEV 1 ), forced vital capacity (FVC) and $F E V_{1} / F V$. DNAme1: DNA methylation at time-point 1; DNAme2: DNA methylation at time-point 2. \#: base model (Mbase) EWAS was covariate adjusted for age, age squared, height, squared deviation from the mean of height, sex and interaction terms of age, age squared, height and squared deviation of height with sex, education llow, medium and high), body mass index, spirometer type, study centre, and cell composition. "ः: smoking model EWAS (Msmok) additionally adjusted for smoking covariates: history of smoking intensity as pack-years smoked up to the time-point of data collection for regressions and for smoking status (current smoker, ex-smoker and never-smoker). EWAS longitudinally predicting the change in lung function (EWASpredict) was additionally adjusted for lung function at time-point 1 .

Cohort-specific EWAS results were summarised by inverse-variance-weighted meta-analyses using METAL [10]. Meta-analysis results were not controlled for genomic inflation after confirming its negligible influence. Epigenome-wide significance level was set to $\mathrm{p}<1 \times 10^{-7}$ (Bonferroni correction, 450000 tests). The selection criteria for replication of sentinel CpGs was less stringent $\left(\mathrm{p}<5 \times 10^{-7}\right)$. Successful replication was defined as a p-value below the outcome-specific Bonferroni correction threshold.

Replicated CpGs were characterised by enrichment, pathway and functional analyses, and additional post hoc analyses were performed (details in supplementary material). 1) A two-sample Mendelian randomisation analysis based on publicly available data was applied to investigate the causality of replicated $\mathrm{CpG}$ associations. 2) A replication of a recently published mediation analysis [4] evidencing 10 smoking-related CpGs mediating the effect of smoking on lung function was undertaken in one discovery cohort (SAPALDIA). 3) To assess the combined effects of smoking-related CpGs on lung function in three discovery cohorts, we built two different DNAme smoking indices based on CpGs: a) predicting lung function effects of smoking [4] and b) located in genome-wide association study (GWAS)-identified lung function genes [2]. These smoking indices were tested for association with lung function in covariate-adjusted linear regression analyses, in all participants and in subgroups stratified by smoking status.

\section{Data availability statement}

Statistical codes and full discovery/replication EWAS effect estimates (meta-analysed and cohort-specific) are made publically available with no end date on the public repository DRYAD (http://datadryad.org/) at the time of publication. Access restrictions apply to the individual methylome data underlying the analysis. Contact details for data requests to the contributing cohorts can be found in the supplementary material.

\section{Results}

Differences in the cohorts' age structure and smoking habits are shown in tables 1 and 2. Mean age was highest for LBC1936 (69.9 years) and youngest for FTC (30.4 years). Self-report of current smoking status 
was lowest in LBC1936 (5.8\%) and highest in LifeLines (43.5\% due to oversampling of current smokers for the DNAme-typed subset).

Across all discovery EWAS meta-analyses, we identified $111 \mathrm{CpG}$ markers for replication $\left(\mathrm{p}<5 \times 10^{-7}: 74\right.$ for FEV1, 16 for FVC and 47 for FEV1/FVC) (supplementary tables S1 and S2). We present here the results for FEV1/FVC (for FEV1 and FVC, refer to the supplementary material).

\section{Cross-sectional associations without smoking adjustment}

In the study-specific and meta-analysed discovery EWAS, the number of lung function-associated DNAme increased from the first to second cross-sectional time-point in the same participants, despite age adjustment (figure $2 \mathrm{a}$ and $\mathrm{b}$ ). We therefore meta-analysed cross-sectional discovery and replication results from the older participants' age time-point available. We observed 29 cross-sectional CpG associations with FEV1/ FVC. 27 of them replicated formally (Bonferroni correction, $\mathrm{p}<0.0011 ; 47$ tests on FEV1/FVC) (table 3 and supplementary table S3). All replicated CpG lung function associations were exclusively DNAme previously associated with smoking [2]. Successful replication was observed for cg05575921 (AHRR), showing the strongest signal for FEV1 and FEV1/FVC (FEV1/FVC: p-value combining discovery and replication cohorts ( pcombined $)=7.22 \times 10^{-50}$ ) among all identified lung function DNAme markers. Methylation at this CpG, previously shown to be hypomethylated with increased smoking, showed positive cross-sectional lung function association. The top $10 \mathrm{CpGs}$ associated with FEV1/FVC (table 3) were located in six loci: cg03636183 (F2RL3), cg21566642, cg01940273 and cg03329539 (vicinity of ALPPL2), cg05575921 and cg21161138 (AHRR), cg23771366 and cg11660018 (PRSS23), cg21611682 (LRP5), and cg15342087 (IER3). The same CpGs, along with cg19572487 (RARA), were also among the top 11 markers cross-sectionally associated with FEV1. Formal replication of cross-sectional associations with FEV1 was observed for 44 CpGs and with FVC for three CpGs (supplementary tables S4 and S5). Similar results were found for repeat cross-sectional analyses (EWASrepeat) (supplementary table S6 and supplementary figure S3).

TABLE 1 Characteristics of discovery cohorts

SAPALDIA 2 time-point 1
SAPALDIA 3 time-point 2
ECRHS II time-point 1
ECRHS III time-point 2

\section{NFBC1966}

(age 31 years) time-point 1
NFBC1966

lage 46 years)

time-point 2

\begin{tabular}{|c|c|c|c|c|c|c|}
\hline Subjects $n$ & 962 & 962 & 470 & 470 & 611 & 611 \\
\hline Female & 53.5 & 53.5 & 56 & 56 & 55.3 & 55.3 \\
\hline Age years & $50.5 \pm 11.3$ & $58.8 \pm 11.3$ & $43.6 \pm 6.8$ & $54.5 \pm 6.8$ & $31.0 \pm 0.3$ & $46.3 \pm 0.4$ \\
\hline Height $\mathrm{cm}$ & $169.4 \pm 9.2$ & $168.7 \pm 9.4$ & $170.0 \pm 9.2$ & $169.2 \pm 9.3$ & $171 \pm 8.8$ & $171 \pm 8.9$ \\
\hline Body mass index $\mathrm{kg} \cdot \mathrm{m}^{-2}$ & $25.8 \pm 4.4$ & $26.5 \pm 4.6$ & $25.0 \pm 4.0$ & $26.5 \pm 4.4$ & $24.2 \pm 3.7$ & $26.7 \pm 4.8$ \\
\hline \multicolumn{7}{|l|}{ Smoking status } \\
\hline Never-smoker ${ }^{\#}$ & 41.7 & 41.1 & 43.2 & 41.7 & 54.5 & 54.5 \\
\hline \multicolumn{7}{|l|}{ Education" } \\
\hline Low & 5.4 & 5.4 & 11.5 & 11.5 & 0.7 & 0.7 \\
\hline Intermediate & 65.7 & 65.7 & 29.2 & 29.2 & 55.9 & 55.9 \\
\hline High & 28.9 & 28.9 & 59.3 & 59.3 & 43.3 & 43.3 \\
\hline $\mathrm{FVC} \mathrm{L}^{+}$ & $4.4 \pm 1.0$ & $4.1 \pm 1.1$ & $4.3 \pm 1.0$ & $3.9 \pm 1.0$ & $4.8 \pm 1.0$ & $4.5 \pm 0.9$ \\
\hline $\mathrm{FEV}_{1} / \mathrm{FVC}<\mathrm{LLN}^{+, \S}$ & 12.9 & 14.1 & 8.7 & 10.4 & 3.3 & 9.5 \\
\hline Doctor-diagnosed asthma & 13.8 & 16.5 & 14.3 & 16.8 & 10.7 & 15.8 \\
\hline Respiratory medication (\% missing values) & $22.2(0.8)$ & $23.7(0.3)$ & 13.4 & 14.2 & NA & NA \\
\hline
\end{tabular}

Data are presented as $\%$ or mean $\pm S D$, unless otherwise stated; percentages may not total $100 \%$ due to rounding. FEV 1 : forced expiratory volume in $1 \mathrm{~s}$; FVC: forced vital capacity; LLN: lower limit of normal; NA: not assessed. "\#: self-reported lifetime nonsmoking. "ः: the categorical variable "education" is defined differently in cohorts (in SAPALDIA low corresponds to primary education; intermediate to secondary, middle or vocational school and high to technical college or university; in ECRHS and NFBC1966 information of age reached at end of studies is used to define low as $\leqslant 16$ years, intermediate as $17-19$ years and high as $\geqslant 20$ years). ${ }^{+}$: values derived from pre-bronchodilation spirometry (lung function values corrected for spirometer device change in SAPALDIA at time-point 2). ${ }^{\S}$ : LLN values estimated using Global Lung Initiative 2012 reference equations [11]. 
TABLE 2 Characteristics of adult replication cohorts

\begin{tabular}{|c|c|c|c|c|c|c|c|}
\hline & $\begin{array}{c}\text { KORA } \\
\text { time-point } 1\end{array}$ & $\begin{array}{c}\text { KORA } \\
\text { time-point } 2\end{array}$ & $\begin{array}{c}\text { LBC1936 } \\
\text { time-point } 1\end{array}$ & $\begin{array}{c}\text { LBC1936 } \\
\text { time-point } 2\end{array}$ & $\begin{array}{c}\text { LifeLines } \\
\text { time-point } 1\end{array}$ & $\begin{array}{c}\text { NSPHS } \\
\text { time-point } 1\end{array}$ & $\begin{array}{c}\text { FTC } \\
\text { time-point } 1\end{array}$ \\
\hline Subjects $n$ & 628 & 628 & 449 & 449 & 1622 & 535 & 93 \\
\hline Age years & $53.6 \pm 4.5$ & $60.1 \pm 4.5$ & $69.6 \pm 0.9$ & $76.3 \pm 0.7$ & $46.7 \pm 10.8$ & $55.1 \pm 16.0$ & $30.4 \pm 3.8$ \\
\hline Height $\mathrm{cm}$ & $169.5 \pm 9.3$ & $168.7 \pm 9.4$ & $167.2 \pm 8.8$ & $166.1 \pm 8.8$ & $176.9 \pm 9.1$ & $163.8 \pm 9.8$ & $173.0 \pm 10.5$ \\
\hline Weight kg & $79.0 \pm 16.7$ & $79.9 \pm 17.3$ & $77.2 \pm 14.6$ & $76.5 \pm 14.8$ & $82.1 \pm 14.7$ & $74.0 \pm 15.2$ & $82.0 \pm 18.8$ \\
\hline Never-smoker $\#$ & 38.2 & 38.2 & 52.3 & 52.3 & 56.6 & 83.2 & 53.8 \\
\hline Ex-smoker & 43.8 & 45.5 & 40.8 & 41.9 & $0^{f}$ & $N A^{\# \#}$ & 26.9 \\
\hline Current smoker & 18.0 & 16.2 & 6.9 & 5.8 & 43.5 & 16.5 & 19.4 \\
\hline Pack-years & $12.8 \pm 19.3$ & $13.5 \pm 20.2$ & $13.9 \pm 24.0$ & $14.1 \pm 24.6$ & $21.0 \pm 11.7$ & $8.1 \pm 21.6$ & NA \\
\hline \multicolumn{8}{|l|}{ Education ${ }^{\pi}$} \\
\hline Low & 47.6 & 47.6 & 49.7 & 49.7 & 23.1 & NA & 1.1 \\
\hline $\mathrm{FEV}_{1} / \mathrm{FVC}^{+}$ & $0.78 \pm 0.06$ & $0.75 \pm 0.07$ & $0.79 \pm 0.09$ & $0.76 \pm 0.12$ & $0.73 \pm 0.09$ & $0.83 \pm 0.09$ & $0.81 \pm 0.07$ \\
\hline \multicolumn{8}{|l|}{ Airflow obstruction } \\
\hline $\mathrm{FEV}_{1} / \mathrm{FVC}<0.7^{+}$ & 8.1 & 20.1 & 15.4 & 26.3 & 38.4 & 8.8 & 5.0 \\
\hline $\mathrm{FEV}_{1} / \mathrm{FVC}<\mathrm{LLN}^{+, \S}$ & 5.0 & 9.6 & 7.6 & 14.9 & 27.5 & 4.3 & 11.3 \\
\hline Doctor-diagnosed asthma & 7.2 & 8.6 & 4.5 & 7.1 & 9.9 & 14.2 & 0 \\
\hline Respiratory medication & 3.3 & 4.9 & 6.7 & 11.8 & 8.0 & 7.7 & 0 \\
\hline
\end{tabular}

Data are presented as $\%$ or mean \pm SD, unless otherwise stated; percentages may not total $100 \%$ due to rounding. FEV 1 : forced expiratory volume in $1 \mathrm{~s}$; FVC: forced vital capacity; LLN: lower limit of normal; NA: not assessed. \#: self-reported lifetime nonsmoking. ": the categorical variable "education" is defined differently in different cohorts. ${ }^{+}$: values derived from pre-bronchodilation spirometry. ${ }^{\S}$ : LLN values estimated using Global Lung Initiative 2012 reference equations [11]. ${ }^{f}$ : LifeLines: nonrandom selection of samples for DNA methylation typing (current smokers versus never-smokers). \#\# : NSPHS: information obtained on current smoking status (yes/no).

Cross-sectional smoking-adjusted associations

The smoking-adjusted EWAS (Msmok) resulted in fewer genome-wide significant results (figure $2 \mathrm{c}$ and $\mathrm{d}$ ). Yet, despite adjustment for self-report of smoking history, the top five CpGs were known smoking-related CpGs. DNAme at cg05575921 (AHRR) remained the top cross-sectional association signal for FEV1/FVC ( pcombined $=2.21 \times 10^{-11}$ ) (supplementary table S7).

Predictive associations without smoking adjustment

The prediction EWAS results (table 4 and figure 3) revealed that DNAme at time-point 1 (DNAme1) at six of nine sentinel CpGs $\left(\mathrm{p}<5 \times 10^{-7}\right)$ associated with change in FEV1/FVC was replicated $(\operatorname{cg} 05575921$ and cg21161138 (AHRR), cg21566642, cg01940273 and cg03329539 (vicinity of ALPPL2), and cg03636183 (F2RL3)). These six replicated CpGs were smoking-related markers. They were also associated with cross-sectional FEV1/FVC and four of them also with predicting change in FEV1 (AHRR (cg05575921), ALPPL2 (cg05951221 and cg01940273) and F2RL3 (cg03636183)) (supplementary table S8).

\section{Associations in never-smokers}

The agnostic discovery EWAS (Mbase) in never-smokers, similar to the entire sample, showed more statistically significant associations at time-point 2 (older age). Eight $\mathrm{CpGs}$ were cross-sectionally associated with FEV1/FVC in never-smokers $\left(\mathrm{p}<5 \times 10^{-7}\right)$, but none replicated (table 5 and supplementary figure S5). The CpG cg14366110 (FIBCD1) showed predictive association of DNAme1 with change in FEV1/FVC ( pdiscovery $=4.2 \times 10^{-9}$, pcombined $=3.6 \times 10^{-9}$ ) in never-smokers, but it did not replicate in KORA and $\mathrm{LBC} 1936$ (preplication $=0.439$; replication cohorts with lung function at two time-points). The direction of effect, however, was consistent (table 6; see supplementary table S9 for cross-sectional associations and supplementary table S10 for prediction associations) in discovery and replication cohorts.

\section{Characterisation of replicated CpGs}

None of the not-smoking-related discovery-identified sentinel CpGs $(n=25)$ were confirmed by replication. In contrast, $78 \%$ of the sentinel CpGs $(n=86)$ had previously been identified as smoking related, and 57 of 

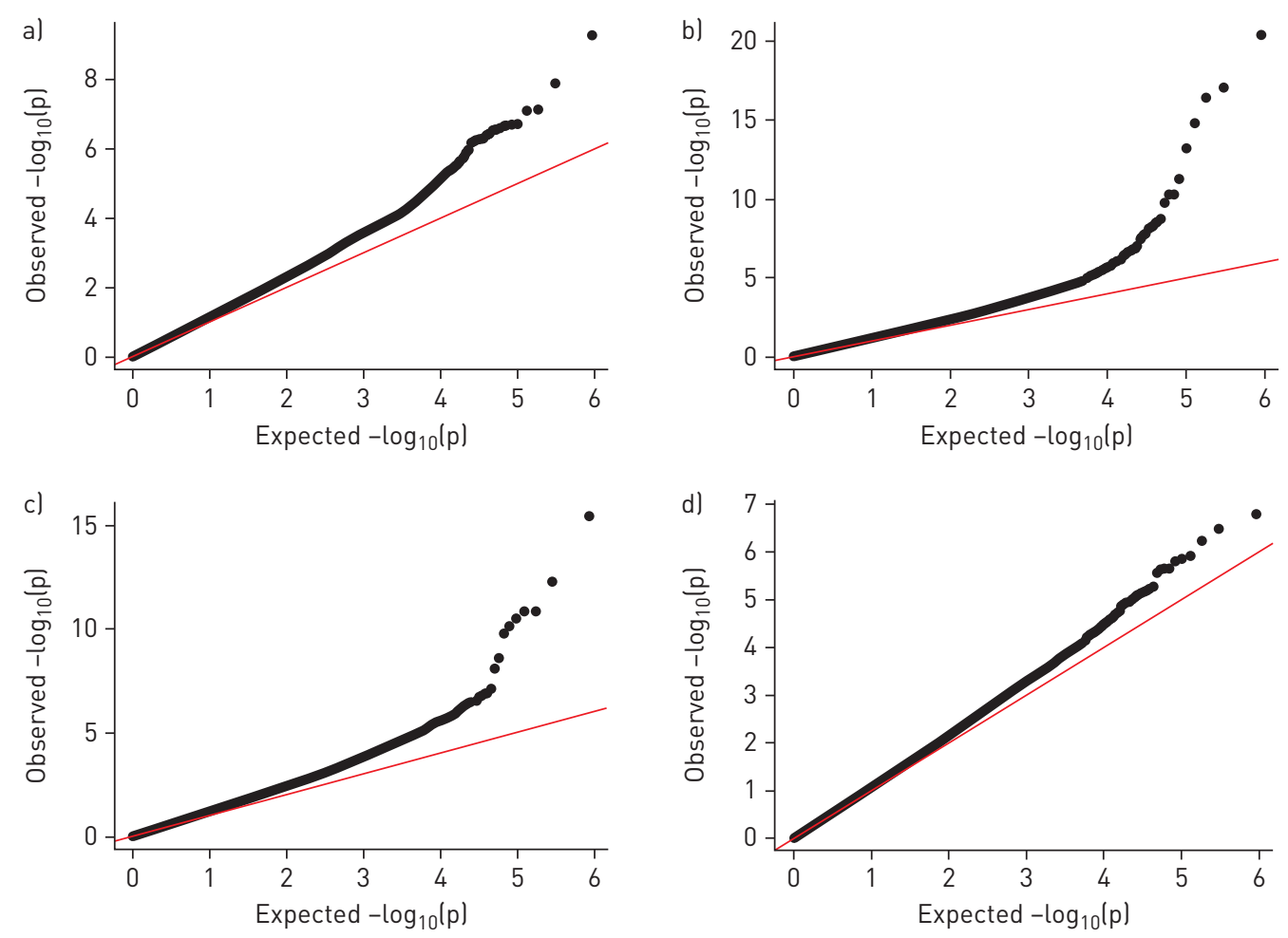

FIGURE 2 a, b) Effect of ageing on the associations between DNA methylation (DNAme) and lung function: quantile-quantile plots of the cross-sectional covariate-adjusted discovery epigenome-wide association study (EWAS) (Mbase ${ }^{\#}$ ) on forced expiratory volume in $1 \mathrm{~s}$ (FEV1)/forced vital capacity (FVC) at a) time-point 1 and b) time-point 2, all participants. Increase in numbers of signals with ageing. For FEV $1 / F V C$, we identified 21 $\mathrm{CpGs}$ at time-point 2 compared with three $\mathrm{CpGs}$ at time-point 1 to be statistically significant. Meta-analyses were performed without genomic control (for time-point 1 inflation factor $\lambda=1.15$ and for time-point 2 inflation factor $\lambda=1.14$ ). For analogous figure for cross-sectional associations with FEV1 and FVC, see supplementary figure S2. c, d) Effect of smoking adjustment on the associations between DNAme and lung function: quantile-quantile plots of $c$ ) the repeat cross-sectional covariate-adjusted discovery EWAS (Mbase ${ }^{\#}$; inflation factor $\lambda=1.13$ ) and $d$ ) additionally smoking adjusted (Msmok"; inflation factor $\lambda=1.05$ ), all participants. Decrease in numbers of signals after smoking adjustment. " : base model (Mbase) EWAS was covariate adjusted for age, age squared, height, squared deviation from the mean of height, sex and interaction terms of age, age squared, height and squared deviation of height with sex, education (low, medium and high), body mass index, spirometer type, study centre, and cell composition. ": smoking-adjusted model (Msmok): covariates applied for Mbase and additionally smoking status and pack-years smoked.

these (mapping to 43 loci) formally replicated across all models and lung function outcomes tested (supplementary table S11). They were used jointly for functional annotation and pathway analyses (supplementary tables S12-S16). Briefly, these 57 lung function-associated CpGs displayed enrichment for transcription factors, such as RELA (false discovery rate-adjusted p-value (pFDR)=0.002) and EP300 $(\mathrm{pFDR}=0.004)$, and suggestive enrichment $(\mathrm{pFDR}<0.1)$ for the chromatin state model of flanking active transcription start sites, of transcription at gene $5^{\prime}$ and $3^{\prime}$, and of enhancers. No significant pathways were revealed using Ingenuity Pathway Analysis database or Gene Ontology term enrichment. Transcriptional misregulation in cancer, pathways in cancer and regulation of actin cytoskeleton were identified ( $\mathrm{pFDR}<0.05$ ) using KEGG (Kyoto Encyclopedia of Genes and Genomes) pathways enrichment.

Using the weighted Kolmogorov-Smirnov test on the entire EWAS discovery results, we noted statistically significant enrichment for smoking-related CpGs among the lung function-associated CpGs. This enrichment was also present in the smoking-adjusted EWAS and even in the EWAS restricted to never-smokers (supplementary table S17).

\section{Association of adult lung function CpG markers with childhood lung function}

Using the same scheme of analysis as for the adult replication cohorts, none of the sentinel CpGs showed associations with FEV1, FVC and FEV1/FVC in the childhood replication cohorts (ALSPAC and IOWBC) (supplementary table S18). The strongest associations observed in children $(\mathrm{p}<0.01)$ were for five CpGs not known to be smoking-related DNAme markers and one smoking-related CpG (cg00310412 (SEMA7A)). 
TABLE 3 Combined epigenome-wide association study (EWAS) meta-analyses of cross-sectional associations ${ }^{\#}$ of $\mathrm{CpG}$ markers with forced expiratory volume in $1 \mathrm{~s}$ (FEV1)/forced vital capacity (FVC) in all participants: base model covariate-adjusted EWAS (Mbase")

\begin{tabular}{|c|c|c|c|c|c|c|c|c|c|c|c|}
\hline CpG & Chr. & $\begin{array}{l}\text { Position } \\
\text { (hg19) }\end{array}$ & Locus & $\beta^{\#} \pm \mathrm{SE}$ & $\begin{array}{c}\text { p-value } \\
\text { meta-analysis }\end{array}$ & $\begin{array}{l}\text { Direction of } \\
\text { effects }^{+}\end{array}$ & $\begin{array}{c}\text { p-value } \\
\text { between-study } \\
\text { heterogeneity }\end{array}$ & $\begin{array}{l}\text { Replicated } \\
p<0.0011^{\S}\end{array}$ & $\begin{array}{l}\text { Previously } \\
\text { reported } \\
\text { smoking } \mathrm{CpG} f\end{array}$ & $\begin{array}{l}\text { Previously } \\
\text { reported } \\
\text { smoking } \\
\text { pFDR-value } f\end{array}$ & $\begin{array}{l}\text { Previously reported } \\
\text { smoking } \\
\text { association } \\
\text { direction of effect }^{f}\end{array}$ \\
\hline cg05575921 & 5 & 373378 & AHRR & $0.124 \pm 0.008$ & $7.22 \times 10^{-50}$ & $+/+/+/+/+/+1+$ & 0.023 & Yes & Yes $^{\# \#}$ & $6.10 \times 10^{-22}$ & $(-)$ \\
\hline cg03636183 & 19 & 17000585 & F2RL3 & $0.201 \pm 0.015$ & $4.50 \times 10^{-43}$ & $+/+1+1+/+1+1+$ & 0.008 & Yes & Yes $\# \#$ & $5.70 \times 10^{-17}$ & $(-1)$ \\
\hline $\operatorname{cg} 21566642$ & 2 & 233284661 & ALPPL2 & $0.151 \pm 0.011$ & $5.02 \times 10^{-43}$ & $+/+/+/+/+/+/+$ & 0.043 & Yes & Yes $\# \#$ & $4.50 \times 10^{-21}$ & $(-)$ \\
\hline cg01940273 & 2 & 233284934 & ALPPL2 & $0.206 \pm 0.015$ & $4.09 \times 10^{-41}$ & $+1+/+1+1+1+1+$ & 0.031 & Yes & Yes $\# \#$ & $9.80 \times 10^{-30}$ & $(-)$ \\
\hline cg03329539 & 2 & 233283329 & ALPPL2 & $0.257 \pm 0.023$ & $5.58 \times 10^{-30}$ & $+/+1+/+1+1+1+$ & 0.628 & Yes & Yes & $9.70 \times 10^{-16}$ & $(-)$ \\
\hline $\operatorname{cg} 21161138$ & 5 & 399360 & AHRR & $0.243 \pm 0.021$ & $9.72 \times 10^{-30}$ & $+/+/+1+/+1+1+$ & 0.152 & Yes & Yes $\# \#$ & $7.90 \times 10^{-13}$ & $(-)$ \\
\hline cg23771366 & 11 & 86510998 & PRSS23 & $0.233 \pm 0.022$ & $5.38 \times 10^{-27}$ & $+/+/+/+/+1+1+$ & 0.286 & Yes & Yes & $1.90 \times 10^{-14}$ & $(-1)$ \\
\hline cg11660018 & 11 & 86510915 & PRSS23 & $0.238 \pm 0.023$ & $3.40 \times 10^{-26}$ & $+/+/+1+1+1+1+$ & 0.318 & Yes & Yes & $4.40 \times 10^{-21}$ & $(-1)$ \\
\hline cg21611682 & 11 & 68138269 & LRP5 & $0.309 \pm 0.030$ & $1.26 \times 10^{-25}$ & $+/+/+1+/+1+1+$ & 0.049 & Yes & Yes & $4.20 \times 10^{-15}$ & $(-1)$ \\
\hline cg15342087 & 6 & 30720209 & IER3 & $0.359 \pm 0.036$ & $5.44 \times 10^{-24}$ & $+/+/+1+/+1+1+$ & 0.169 & Yes & Yes & $3.90 \times 10^{-14}$ & $(-)$ \\
\hline $\operatorname{cg} 26703534$ & 5 & 377358 & $A H R R$ & $0.266 \pm 0.026$ & $7.34 \times 10^{-24}$ & $+/+1+/+1+1+1+$ & 0.101 & Yes & Yes & $7.20 \times 10^{-18}$ & $(-)$ \\
\hline $\operatorname{cg} 25648203$ & 5 & 395444 & $A H R R$ & $0.250 \pm 0.026$ & $9.84 \times 10^{-22}$ & $+/+/+1+/+1+/+$ & 0.194 & Yes & Yes & $2.70 \times 10^{-11}$ & $(-)$ \\
\hline cg19572487 & 17 & 38476024 & RARA & $0.196 \pm 0.021$ & $8.87 \times 10^{-21}$ & $+/+/+1+/+/+1+$ & 0.018 & Yes & Yes & $1.60 \times 10^{-16}$ & $(-1)$ \\
\hline cg00310412 & 15 & 74724918 & SEMA7A & $0.261 \pm 0.028$ & $4.01 \times 10^{-20}$ & $+/+1+/+1+/+1+$ & 0.275 & Yes & Yes & $1.20 \times 10^{-13}$ & $1-1$ \\
\hline $\operatorname{cg} 24859433$ & 6 & 30720203 & IER3 & $0.303 \pm 0.034$ & $2.05 \times 10^{-19}$ & $+/+/+/+/+/+1+$ & 0.067 & Yes & Yes $^{\# \#}$ & $2.20 \times 10^{-9}$ & $(-1)$ \\
\hline cg09935388 & 1 & 92947588 & GFI1 & $0.105 \pm 0.012$ & $7.05 \times 10^{-19}$ & $+/+/+1+/+1+1+$ & 0.034 & Yes & Yes $^{\# \#}$ & $7.00 \times 10^{-14}$ & $(-1)$ \\
\hline cg14753356 & 6 & 30720108 & IER3 & $0.189 \pm 0.021$ & $9.08 \times 10^{-19}$ & $+/+/+/+/+1+1+$ & 0.405 & Yes & Yes & $2.30 \times 10^{-14}$ & $1-1$ \\
\hline cg04885881 & 1 & 11123118 & $\begin{array}{c}\text { SRM/ } \\
\text { EXOSC10 }\end{array}$ & $0.168 \pm 0.020$ & $5.66 \times 10^{-18}$ & $+/+/+/+/+/+/+$ & 0.670 & Yes & Yes & $2.70 \times 10^{-11}$ & $(-)$ \\
\hline $\operatorname{cg} 25949550$ & 7 & 145814306 & CNTNAP2 & $0.335 \pm 0.039$ & $6.04 \times 10^{-18}$ & $+/+/+/+/+1+/+$ & 0.013 & Yes & Yes & $9.30 \times 10^{-21}$ & $(-)$ \\
\hline $\operatorname{cg} 19859270$ & 3 & 98251294 & GPR15 & $0.467 \pm 0.055$ & $2.80 \times 10^{-17}$ & $+/+/+/+1+1+1+$ & 0.029 & Yes & Yes & $6.30 \times 10^{-17}$ & $(-)$ \\
\hline $\operatorname{cg} 03450842$ & 10 & 80834947 & ZMIZ1 & $0.265 \pm 0.031$ & $2.92 \times 10^{-17}$ & $+/+/+/+/+/+/+$ & 0.003 & Yes & Yes & $2.40 \times 10^{-11}$ & $(-)$ \\
\hline cg03707168 & 19 & 49379127 & PPP1R15A & $0.206 \pm 0.025$ & $1.27 \times 10^{-16}$ & $+/+/+/+/+/+1+$ & 0.668 & Yes & Yes & $3.50 \times 10^{-7}$ & $(-1)$ \\
\hline cg17087741 & 2 & 233283010 & ALPPL2 & $0.161 \pm 0.020$ & $4.48 \times 10^{-16}$ & $+1+1+1+1+1+1-$ & $<0.001$ & Yes & Yes & $6.10 \times 10^{-7}$ & $(-1)$ \\
\hline cg21140898 & 1 & 51442318 & CDKN2C & $0.120 \pm 0.017$ & $4.46 \times 10^{-13}$ & $+/+/+/+/+/+/+$ & 0.103 & Yes & Yes & $3.70 \times 10^{-8}$ & $i-1$ \\
\hline cg01899089 & 5 & 369969 & $A H R R$ & $0.172 \pm 0.027$ & $1.47 \times 10^{-10}$ & $+/+/+/+/+/+/+$ & 0.005 & Yes & Yes & $1.80 \times 10^{-12}$ & $1-1$ \\
\hline cg08763102 & 4 & 3079751 & HTT & $0.225 \pm 0.039$ & $1.20 \times 10^{-8}$ & $+/+/+/+1+1+1-$ & 0.001 & Yes & Yes & $3.80 \times 10^{-15}$ & $1-1$ \\
\hline cg21282907 & 6 & 74289980 & SLC17A5 & $0.176 \pm 0.031$ & $1.28 \times 10^{-8}$ & $+1+1+1+1+1+1-$ & 0.003 & No & Yes & $1.28 \times 10^{-2}$ & $(-1)$ \\
\hline cg20853880 & 2 & 10184444 & KLF11 & $0.077 \pm 0.014$ & $6.05 \times 10^{-8}$ & 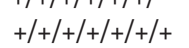 & 0.052 & No & Yes & $3.70 \times 10^{-7}$ & $(-1)$ \\
\hline cg16391678 & 16 & 30485597 & ITGAL & $0.164 \pm 0.031$ & $1.15 \times 10^{-7}$ & $+1+1+1+1+1+1-$ & 0.003 & Yes & Yes & $3.00 \times 10^{-11}$ & $(-1)$ \\
\hline
\end{tabular}

Chr.: chromosome; hg19: human genome build 19; $\beta$ : coefficient of association; FDR: false discovery rate. Meta-analyses of cross-sectional associations obtained using data from the oldest time-point available: time-point 2 of ECRHS, NFBC1966, SAPALDIA and LBC1936; time-point 1 of KORA, LifeLines and NSPHS. For complete results for FEV1/FVC associations,

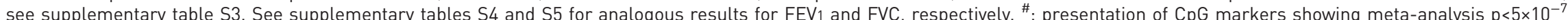
in the combined meta-analysis. Note that DNA methylation predictors used were technical bias-adjusted, normalised residuals and thus effect sizes of the association ( $\beta$ ) are not directly comparable to effect sizes reported elsewhere using normalised \% methylation as predictor. ๆ: base model (Mbase) epigenome-wide association study was covariate adjusted for age, age squared, height, squared deviation from the mean of height, sex and interaction terms of age, age squared, height and squared deviation of height with sex, education (low, medium and high), body mass index, spirometer type, study centre as well as cell composition. : order of cohorts: ECRHS, NFBC1966, SAPALDIA, KORA, LBC1936, LifeLines and NSPHS (FTC was excluded from this meta-analysis, given the smaller sample size and lower mean age (30.4 years) compared with the other adult cohorts (ECRHS (54.5 years), NFBC1966 ( 46.3 years), SAPALDIA (58.8 years) and LBC1936 (76.3 years), and the single available time-point for KORA (60.1 years), LifeLines (46.7 years) and NSPHS (55.1 years)! \$. replication was defined for association if replication $p<0.0011$ (multiple testing correction, 47 tests for FEV1/FVC). ${ }^{f}$ : smoking CpGs defined on the reported FDR-corrected p<0.05 for association reported with smoking status and reported direction of effects for association with smoking [2]. \#\#: smoking CpG previously reported to mediate the effect of smoking on lung function [4]. 
TABLE 4 Combined meta-analyses of the prediction associations $\#$ of $\mathrm{CpG}$ markers on annual change in forced expiratory volume in $1 \mathrm{~s}$ (FEV 1 )/forced vital capacity (FVC) in all participants: base model adjustment (Mbase")

\begin{tabular}{|c|c|c|c|c|c|c|c|c|c|c|c|}
\hline \multirow[t]{2}{*}{ CpG } & \multirow[t]{2}{*}{ Chr. } & \multirow[t]{2}{*}{$\begin{array}{l}\text { Position } \\
\text { (hg19) }\end{array}$} & \multirow[t]{2}{*}{ Locus } & \multicolumn{5}{|c|}{$\begin{array}{c}\text { Combined meta-analysis } \\
\text { (ECRHS/NFBC1966/SAPALDIA/KORA/LBC1936) }\end{array}$} & \multirow{2}{*}{$\begin{array}{l}\text { Previously } \\
\text { reported } \\
\text { smoking } \mathrm{CpG}^{f}\end{array}$} & \multirow{2}{*}{$\begin{array}{l}\text { Previously } \\
\text { reported } \\
\text { smoking } \\
\text { pFDR-value }\end{array}$} & \multirow{2}{*}{$\begin{array}{l}\text { Previously reported } \\
\text { smoking association } \\
\text { direction of effect }^{f}\end{array}$} \\
\hline & & & & $\beta^{\#} \pm$ SE & $\begin{array}{c}\text { p-value } \\
\text { meta-analysis }\end{array}$ & $\begin{array}{l}\text { Direction of } \\
\text { effects }^{+}\end{array}$ & $\begin{array}{c}\text { p-value } \\
\text { between-study } \\
\text { heterogeneity }\end{array}$ & $\begin{array}{l}\text { Replicated } \\
p<0.0011^{\S}\end{array}$ & & & \\
\hline cg05575921 & 5 & 373378 & $A H R R$ & $0.006 \pm 0.001$ & $2.77 \times 10^{-13}$ & $+/+/+/+/+$ & 0.005 & Yes & Yes ${ }^{\# \#}$ & $6.10 \times 10^{-22}$ & $(-)$ \\
\hline cg21566642 & 2 & 233284661 & ALPPL2 & $0.006 \pm 0.001$ & $3.17 \times 10^{-11}$ & $+1+1+1+1+$ & 0.235 & Yes & Yes ${ }^{\# \#}$ & $4.50 \times 10^{-21}$ & $(-)$ \\
\hline cg01940273 & 2 & 233284934 & ALPPL2 & $0.009 \pm 0.001$ & $4.93 \times 10^{-11}$ & $+1+1+1+1+$ & 0.023 & Yes & Yes $^{\# \#}$ & $9.80 \times 10^{-30}$ & $(-)$ \\
\hline cg21161138 & 5 & 399360 & $A H R R$ & $0.011 \pm 0.002$ & $5.81 \times 10^{-9}$ & $+1+1+1+1+$ & 0.103 & Yes & Yes $^{\# \#}$ & $7.90 \times 10^{-13}$ & $(-)$ \\
\hline cg03636183 & 19 & 17000585 & F2RL3 & $0.008 \pm 0.001$ & $6.22 \times 10^{-9}$ & $+1+1+1+1+$ & 0.001 & Yes & Yes ${ }^{\# \#}$ & $5.70 \times 10^{-17}$ & $(-)$ \\
\hline cg01377124 & 2 & 237172609 & ASB18 & $-0.018 \pm 0.003$ & $7.38 \times 10^{-8}$ & $-1-1+1-1+$ & 0.005 & No & No & NA & NA \\
\hline cg03329539 & 2 & 233283329 & ALPPL2 & $0.011 \pm 0.002$ & $7.66 \times 10^{-8}$ & $+1+/+1+1+$ & 0.015 & Yes & Yes & $9.70 \times 10^{-16}$ & $(-)$ \\
\hline cg07222133 & 5 & 179499488 & RNF130 & $-0.009 \pm 0.002$ & $2.45 \times 10^{-7}$ & $? /-/+/-/+$ & $<0.001$ & No & No & NA & NA \\
\hline $\operatorname{cg} 14366110$ & 9 & 133779382 & FIBCD1 & $0.014 \pm 0.003$ & $9.62 \times 10^{-7}$ & $+1+1+1-1-$ & 0.206 & No & No & NA & NA \\
\hline
\end{tabular}

Chr.: chromosome; hg19: human genome build 19; $\beta$ : coefficient of association; FDR: false discovery rate; NA: not assessed. For complete results for FEV1/FVC and analogous results for FEV1 and FVC, see supplementary table S8. \#: predictive associations of DNA methylation at first time-point (DNAme1) with annual change in lung function during follow-up, defined as (lung function at second time-point-lung function at first time-point)/time of follow-up (years). Presentation of $\mathrm{CpG}$ markers showing meta-analysis $p$-value $<5 \times 10^{-7}$ at discovery or combined meta-analyses level. CpGs shown sorted by statistical significance of combined meta-analysis results. Note that DNAme predictors used were technical bias-adjusted, normalised residuals and thus effect sizes of the association ( $\beta$ ) are not directly comparable to effect sizes reported elsewhere using normalised \% methylation as predictor. ": base model $\left(M_{\text {base }}\right)$ epigenome-wide association study was covariate adjusted for age, age squared, height, FEV/FVC at time-point 1 , squared deviation from the mean of height, sex and interaction terms of age, age squared, height and squared deviation of height with sex, education (low, medium and high), body mass index, spirometer type, study centre as well as cell composition. ${ }^{+}$: order of cohorts: ECRHS, NFBC1966, SAPALDIA, KORA and LBC1936. §: replication was defined for association if replication p<0.0011 (multiple testing correction, 47 tests for $\mathrm{FEV}_{1} / \mathrm{FVC}$ ]. ${ }^{f}$ : smoking $\mathrm{CpGs}$ defined on the reported FDR-corrected $\mathrm{p}<0.05$ for association reported with smoking status and reported direction of effects for association with smoking [2]. \#\#: smoking $\mathrm{CpG}$ previously reported to mediate the effect of smoking on lung function [4]. 

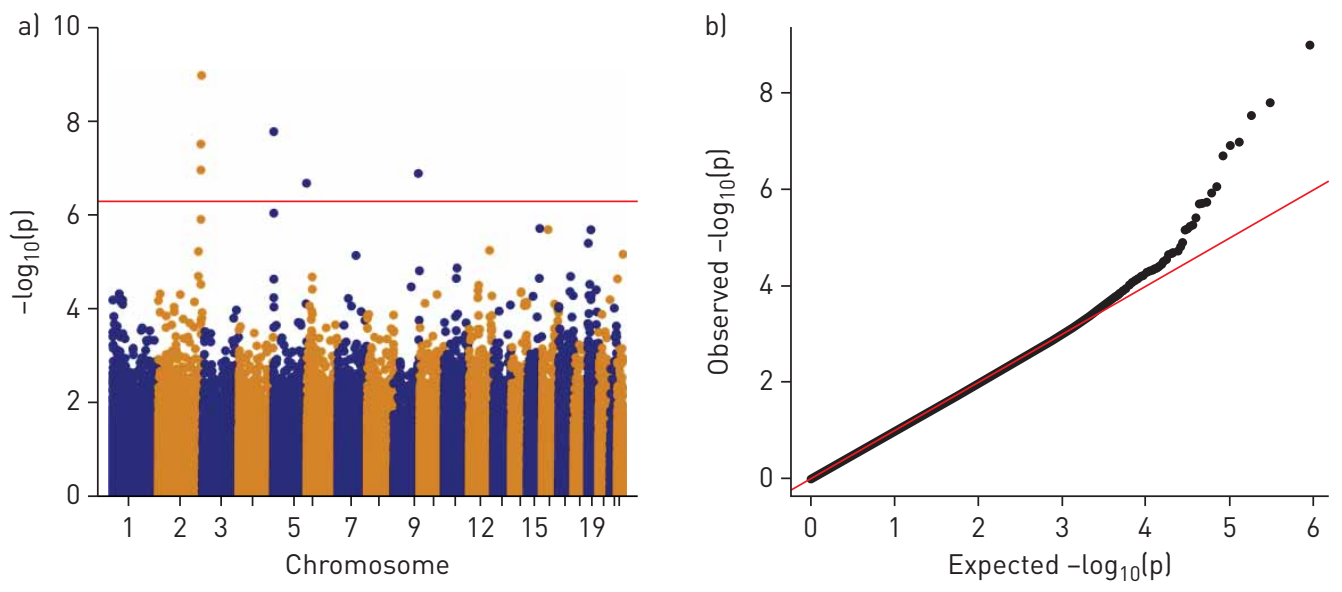

FIGURE 3 a) Manhattan and b) quantile-quantile plots of the covariate-adjusted prediction ${ }^{\#}$ epigenome-wide association study (EWAS) (Mbase") on forced expiratory volume in $1 \mathrm{~s}$ (FEV 1 )/forced vital capacity (FVC), all participants. Meta-analysis of the prediction association was performed without genomic control (inflation factor $\lambda=0.95$ ). For analogous figure for associations with change in FEV1 and FVC, see supplementary figure S4. \#: predictive associations of DNA methylation at first time-point with change in lung function during follow-up. ๆ: base model (Mbase) EWAS was covariate adjusted for age, age squared, height, FEV1/FVC at time-point 1, squared deviation from the mean of height, sex and interaction terms of age, age squared, height and squared deviation of height with sex, education (low, medium and high), body mass index, spirometer type, study centre, and cell composition.

\section{Comparison with published DNAme-lung function association reports}

Our agnostic results were compared with previously reported lung function-specific $[4,6,7,12]$ or chronic obstructive pulmonary disease (COPD)-specific $[13,14]$ DNAme. We retrieved all CpGs reported being associated with lung function $(\mathrm{n}=376)$ for a look-up in the cross-sectional FEV1, FVC and FEV1/FVC associations at time-point 2. Only 12 out of 376 CpGs showed evidence for association (Bonferroni correction for 376 tests: $\mathrm{p}<1.3 \times 10^{-4}$ ) (supplementary table S19). Notably, the most recently reported CpG markers $[4,6]$, having also been related to smoking, showed consistent associations with lung function, e.g. cg05575921 and cg21161138 (AHRR), cg05951221 (near ALPPL2), and cg06126421 (IER3). They were among our top replicated lung function association signals.

\section{Two-sample Mendelian randomisation investigation}

To assess the causality of replicated DNAme-lung function association, we conducted a post hoc Mendelian randomisation look-up using publicly available databases $[15,16]$. Genetic instruments were identified for 12 replicated CpGs. A two-sample Mendelian randomisation on cross-sectional lung function could be completed for seven CpGs (supplementary table S20). Results support causal effects for cg23771366 and cg11660018 (PRSS23), cg21990700 (C1RL), and cg00073460 (ZC3H12D) on FEV1, and for cg00073460 (ZC3H12D) and cg24086068 (SHROOM3) on FVC.

\section{Integration of DNAme into a smoking index}

A recent smoking EWAS followed-up by a mediation analysis identified $10 \mathrm{CpGs}$ as mediators of the smoking-lung function association [4]. Eight of these mediating CpGs were among our replicated lung function-associated CpGs (supplementary table S21). In a post hoc mediation analysis in SAPALDIA, we showed statistically significant average causal mediation on lung function for nine of these mediating CpGs (FEV1/FVC: table 7; FEV1 and FVC: supplementary table S22).

To assess the combined effect of these smoking exposure-mediating $\mathrm{CpGs}$ on lung function, we constructed a mediation smoking index (Mediation-SI). Its association with lung function by smoking status was tested in covariate-adjusted regression models in the discovery cohorts and following EWAS models (SAPALDIA, ECRHS and NFBC1966). Meta-analysed results of Mediation-SI showed strong association with cross-sectional FEV1/FVC in all participants and ever-smokers (table 8 and figure 4; FEV1 and FVC: supplementary table S23). Mediation-SI association in all participants was more pronounced for crosssectional $\left(\beta \pm S E-1.2 \pm 0.13 ; p=2.65 \times 10^{-20}\right)$ than for prediction association $(\beta \pm S E-0.03 \pm 0.01 ; p=0.0072)$. We noted comparable associations of Mediation-SI and of pack-years with lung function (figure 5). Both were inversely associated with level of FEV1/FVC. Adding Mediation-SI or self-reported smoking history (smoking status and pack-years) to the different Mbase-adjusted statistical models showed a comparable increase in total adjusted $\mathrm{R}^{2}$. The highest total adjusted $\mathrm{R}^{2}$ was obtained when including both DNAme 
TABLE 5 Combined meta-analyses ${ }^{\#}$ of cross-sectional associations on forced expiratory volume in $1 \mathrm{~s}$ (FEV1)/forced vital capacity (FVC) in never-smokers only: base model adjustment (Mbase?)

CpG

Chr. Position Locus
(hg19)

Locus

Combined meta-analysis
(ECRHS/NFBC1966/SAPALDIA/KORA/LBC193

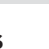

$\beta^{\#} \pm \mathrm{SE}$ $\mathrm{p}$-value
meta-analysis

Direction of
effects $^{+}$

p-value tween-study heterogeneity

PHS)

licated

\section{Previously \\ reported}

smoking $\mathrm{CpG}^{f}$

\begin{tabular}{ccccc}
\hline cg09884077 & 15 & 23086698 & NIPA1 & $-0.308 \pm 0.084$ \\
cg25758394 & 1 & 3623859 & TP73 & $0.213 \pm 0.083$ \\
cg18664508 & 3 & 169487465 & ARPM1 & $-0.308 \pm 0.072$ \\
cg19268386 & 15 & 23086595 & NIPA1 & $-0.263 \pm 0.140$ \\
cg15981995 & 3 & 169487311 & ARPM1 & $-0.231 \pm 0.073$ \\
cg05785298 & 1 & 204654622 & LRRN2 & $-0.423 \pm 0.111$ \\
cg20278790 & 20 & 57583474 & CTSZ & $0.319 \pm 0.070$ \\
cg13562246 & 8 & 33368277 & C8orf41 & $0.349 \pm 0.074$
\end{tabular}

\begin{abstract}
$-1-1-1+1-1-1-$
? $/ 2+1-1-1+1-$

$+1-1-1-1-1-1-$

$2 / 2 /-1-1-1-$
$+1-1-1-1-1-1$

?/?-1-1-1-/+

$-1+1-1+1-1+1-$

$-1+1+1+1-1-1-$

$+/+/+/+/+/-/+$
\end{abstract}

0.001
$<0.001$
$<0.001$
$<0.001$
$<0.001$
0.001
$<0.001$
0.206

No

No

No

No

No

No

No

No

$\begin{array}{r}\text { Previo } \\ \text { repor } \\ \text { smok } \\ \text { pFDR-v }\end{array}$
NA
NA
NA
NA
NA
NA
NA
NA

Chr.: chromosome; hg19: human genome build 19; $\beta$ : coefficient of association; FDR: false discovery rate; NA: not assessed. For complete results for FEV1/FVC and for FEV1 and FVC in never-smokers, see supplementary table S8. \#: presentation of $\mathrm{CpG}$ markers showing meta-analysis $p<5 \times 10^{-7}$ at discovery level for cross-sectional association at time-point 2 , using data from time-point 2 of ECRHS, NFBC1966, SAPALDIA and LBC1936 and from time-point 1 of KORA, LifeLines and NSPHS. Note that DNA methylation predictors used were technical bias-adjusted, normalised residuals and thus effect sizes of the association ( $\beta$ ) are not directly comparable to effect sizes reported elsewhere using normalised \% methylation as predictor. ": base model (Mbase) epigenome-wide association study was covariate adjusted for age, age squared, height, squared deviation from the mean of height, sex and interaction terms of age, age squared, height and squared deviation of height with sex, education (low, medium and high), body mass index, spirometer type, study centre as well as cell composition. ${ }^{+}$: order of cohorts: ECRHS, NFBC1966, SAPALDIA, KORA, LBC1936, LifeLines and NSPHS. \$: replication was defined for association if replication p<0.0011 (multiple testing correction, 47 tests for $\left.F E V_{1} / F V C\right) .{ }^{f}$ : smoking $\mathrm{CpGs}$ defined on the reported FDR-corrected $p<0.05$ for association reported with smoking status and reported direction of effects for association with smoking [2].

TABLE 6 Combined meta-analyses of the prediction associations ${ }^{\#}$ of $\mathrm{CpG}$ markers on annual change in forced expiratory volume in $1 \mathrm{~s}$ (FEV 1 )/forced vital capacity (FVC) in never-smokers only: base model adjustment (Mbase ${ }^{\text {I) }}$

\begin{tabular}{|c|c|c|c|c|c|c|c|c|c|c|c|}
\hline \multirow[t]{2}{*}{ CpG } & \multirow[t]{2}{*}{ Chr. } & \multirow[t]{2}{*}{$\begin{array}{l}\text { Position } \\
\text { (hg19) }\end{array}$} & \multirow[t]{2}{*}{ Locus } & \multicolumn{5}{|c|}{$\begin{array}{c}\text { Combined meta-analysis } \\
\text { (ECRHS/NFBC1966/SAPALDIA/KORA/LBC1936) }\end{array}$} & \multirow{2}{*}{$\begin{array}{l}\text { Previously } \\
\text { reported } \\
\text { smoking CpG } f\end{array}$} & \multirow{2}{*}{$\begin{array}{l}\text { Previously } \\
\text { reported } \\
\text { smoking } \\
\text { pFDR-value }\end{array}$} & \multirow{2}{*}{$\begin{array}{l}\text { Previously reported } \\
\text { smoking association } \\
\text { direction of effect }\end{array}$} \\
\hline & & & & $\beta^{\#} \pm \mathrm{SE}$ & $\begin{array}{c}\text { p-value } \\
\text { meta-analysis }\end{array}$ & $\begin{array}{l}\text { Direction of } \\
\text { effects }^{+}\end{array}$ & $\begin{array}{c}\text { p-value } \\
\text { between-study } \\
\text { heterogeneity }\end{array}$ & $\begin{array}{l}\text { Replicated } \\
p<0.0011^{\S}\end{array}$ & & & \\
\hline $\operatorname{cg} 14366110$ & 9 & 133779382 & FIBCD1 & $0.017 \pm 0.003$ & $3.60 \times 10^{-9}$ & $+/+/-1+/+$ & 0.315 & No & No & NA & NA \\
\hline cg11216682 & 2 & 131113867 & PTPN18 & $-0.017 \pm 0.003$ & $1.10 \times 10^{-7}$ & $+1-1+1-1-$ & 0.282 & No & No & NA & NA \\
\hline
\end{tabular}

Chr.: chromosome; hg19: human genome build 19; $\beta$ : coefficient of association; FDR: false discovery rate; NA: not assessed. For complete results for FEV1/FVC and for analogous results for FEV1 and FVC, see supplementary table S9. \#: predictive associations of DNA methylation at first time-point (DNAme1) with annual change in lung function during follow-up, defined as (lung function at second time-point-lung function at first time-point)/time of follow-up (years). Presentation of $\mathrm{CpG}$ markers showing meta-analysis $\mathrm{p}<5 \times 10^{-7}$ at discovery or replication level. Note that DNAme predictors used were technical bias-adjusted, normalised residuals and thus effect sizes of the association ( $\beta$ ) are not directly comparable to effect sizes reported elsewhere using normalised \% methylation as predictor. ": base model (Mbase) epigenome-wide association study was covariate adjusted for age, age squared, height, FEV1/FVC at time-point 1, squared deviation from the mean of height, sex and interaction terms of age, age squared, height and squared deviation of height with sex, education (low, medium and high), body mass index, spirometer type, study centre as well as cell composition. ${ }^{+}$: order of cohorts: ECRHS, NFBC1966, SAPALDIA, KORA and LBC1936. ${ }^{\S}$ : replication was defined for association if replication $\mathrm{p}<0.0011$ (multiple testing correction, 47 tests for FEV1/FVC). ${ }^{f}$ : smoking CpGs defined on the reported FDR-corrected $p<0.05$ for association reported with smoking status and reported direction of effects for association with smoking [2]. 
TABLE 7 Mediation" analysis on the role of previously reported CpGs in the smoking association with forced expiratory volume in $1 \mathrm{~s}$ (FEV1)/forced vital capacity (FVC): the SAPALDIA cohort

\begin{tabular}{|c|c|c|c|c|c|c|c|c|c|}
\hline \multirow[t]{2}{*}{$\mathrm{CpG}^{\pi}$} & \multirow[t]{2}{*}{ Locus } & \multicolumn{2}{|l|}{ ACME } & \multicolumn{2}{|l|}{ ADE } & \multicolumn{2}{|l|}{ Total effect } & \multicolumn{2}{|l|}{ Proportion } \\
\hline & & Estimate $(95 \% \mathrm{CI})$ & $p$-value & Estimate $(95 \% \mathrm{CI})$ & p-value & Estimate $(95 \% \mathrm{CI})$ & $\mathrm{p}$-value & Estimate $(95 \% \mathrm{CI})$ & p-value \\
\hline cg01940273 & ALPPL2 & $-0.0079(-0.0119--0.0041)$ & $<0.0001$ & $-0.0026(-0.0129-0.0077)$ & 0.604 & $-0.0106(-0.0203--0.0014)$ & 0.026 & $0.7313(0.2616-3.4325)$ & 0.026 \\
\hline cg03636183 & F2RL3 & $-0.0080(-0.0122--0.0040)$ & $<0.0001$ & $-0.0029(-0.0126-0.0062)$ & 0.556 & $-0.0108(-0.0197--0.0021)$ & 0.018 & 0.7312 (0.2819-2.9097) & 0.018 \\
\hline cg05575921 & $A H R R$ & $-0.0102(-0.0147--0.0055)$ & $<0.0001$ & $-0.0008(-0.0109-0.0086)$ & 0.870 & $-0.0110(-0.0202--0.0020)$ & 0.012 & 0.9213 (0.3818-4.0453) & 0.012 \\
\hline cg05951221 & ALPPL2 & $-0.0075(-0.0122--0.0030)$ & 0.002 & $-0.0033(-0.0131-0.0062)$ & 0.520 & $-0.0109(-0.0197--0.0022)$ & 0.020 & $0.6836(0.1942-2.7656)$ & 0.022 \\
\hline cg06126421 & IER3 & $-0.0054(-0.0093--0.0017)$ & $<0.0001$ & $-0.0049(-0.0148-0.0049)$ & 0.328 & $-0.0103(-0.0194--0.0012)$ & 0.030 & $0.5233(0.1050-2.5558)$ & 0.030 \\
\hline cg09935388 & GF/1 & $-0.0033(-0.0058--0.0010)$ & 0.002 & $-0.0073(-0.0168-0.0022)$ & 0.122 & $-0.0105(-0.0198--0.0013)$ & 0.034 & 0.3009 (0.0568-1.4190) & 0.036 \\
\hline $\operatorname{cg} 21161138$ & $A H R R$ & $-0.0056(-0.0089--0.0025)$ & $<0.0001$ & $-0.0052(-0.0146-0.0043)$ & 0.282 & $-0.0108(-0.0194--0.0020)$ & 0.020 & $0.5127(0.1647-2.0961)$ & 0.020 \\
\hline cg21566642 & ALPPL2 & $-0.0098(-0.0145--0.0057)$ & $<0.0001$ & $-0.0014(-0.0116-0.0089)$ & 0.796 & $-0.0112(-0.0209--0.0011)$ & 0.024 & $0.8663(0.3453-4.6567)$ & 0.024 \\
\hline $\operatorname{cg} 22994830$ & PRKAR1B & $-0.0002(-0.0009-0.0003)$ & 0.542 & $-0.0103(-0.0201--0.0010)$ & 0.028 & $-0.0105(-0.0202--0.0013)$ & 0.024 & $0.0103(-0.0470-0.1595)$ & 0.550 \\
\hline $\operatorname{cg} 24859433$ & IER3 & $-0.0024(-0.0053-0.0002)$ & 0.068 & $-0.0082(-0.0179-0.0013)$ & 0.112 & $-0.0107(-0.0201--0.0014)$ & 0.022 & $0.2186(-0.0438-1.2776)$ & 0.090 \\
\hline
\end{tabular}

ACME: average causal mediation effect; ADE: average direct effect. For analogous results for FEV1 and FVC, see supplementary table S22. ${ }^{\#}$ : performed using the R package mediation [17]. I: previously reported candidate $\mathrm{CpG}$ for mediation of effect of smoking on lung function [4]. 
TABLE 8 Meta-analyses ${ }^{\#}$ of the discovery cohort-specific association of mediation smoking index (Mediation-SI) with forced expiratory volume in $1 \mathrm{~s}(\mathrm{FEV} 1) /$ forced vital capacity (FVC) (\%), cross-sectionally at time-point 2 and longitudinally predicting the annual change during follow-up, in all study participants, ever and never-smokers: base model adjustment (Mbase")

Cross-sectional meta-analysis at time-point $2^{\#}$

$\begin{array}{llcc}\beta \pm \mathrm{SE} & \mathrm{p}-\text { value }^{+} & \begin{array}{c}\text { Direction } \\ \text { of effects }\end{array} & \begin{array}{c}\text { p-value } \\ \text { between-study } \\ \text { heterogeneity }\end{array}\end{array}$

Prediction on change in lung function"

$\begin{array}{llcc}\beta \pm S E & p-\text { value }^{+} & \begin{array}{c}\text { Direction } \\ \text { of effects }\end{array} & \begin{array}{c}\text { p-value } \\ \text { between-study } \\ \text { heterogeneity }\end{array}\end{array}$

\begin{tabular}{|c|c|c|c|c|c|c|c|c|}
\hline All & $-0.012 \pm 0.0013$ & $1.05 \times 10^{-20}$ & $-1-1-$ & 0.44 & $-0.0005 \pm 0.0001$ & $8.66 \times 10^{-9}$ & $-1-1-$ & 0.006 \\
\hline Ever-smokers & $-0.014 \pm 0.0016$ & $3.28 \times 10^{-18}$ & $-1-1-$ & 0.30 & $-0.0004 \pm 0.0001$ & $4.94 \times 10^{-4}$ & $-1-1-$ & 0.13 \\
\hline Never-smokers & $-0.0033 \pm 0.0041$ & 0.423 & $-1-1+$ & 0.62 & $-0.0007 \pm 0.0002$ & $1.73 \times 10^{-4}$ & $+/-/+$ & 0.003 \\
\hline
\end{tabular}

$\beta$ : coefficient of association. For analogous results of associations of Mediation-SI with FEV1 and FVC, see supplementary table S23. \#: cohort-specific association results for Mediation-SI were meta-analysed. The 10 CpGs contributing Mediation-SI values are shown in supplementary table S21. Note that DNA methylation predictors used were technical bias-adjusted, normalised residuals and thus effect sizes of the association $(\beta)$ are not directly comparable to effect sizes reported elsewhere using normalised \% methylation as predictor. १: base model (Mbase) covariate adjustment: age, age squared, height, squared deviation from the mean of height, sex and interaction terms of age, age squared, height and squared deviation of height with sex, education (low, medium and high), body mass index, spirometer type, study centre as well as cell composition. Prediction models were additionally adjusted for FEV1/FVC at time-point $1 .{ }^{+}$: $p$-value of meta-analysis: $p<0.008$ was considered statistically significant, Bonferroni correction for six tests per lung function outcome. ${ }^{\S}$ : order of cohorts: ECRHS, NFBC1966 and SAPALDIA.

score and self-reported smoking history. Covariate-adjusted mean Mediation-SI values decreased from never- to ex- to current smokers and from more distant to more recent smoking exposure, with increase in pack-years in current smokers and with fewer years since quitting in ex-smokers (figure 6).

a)

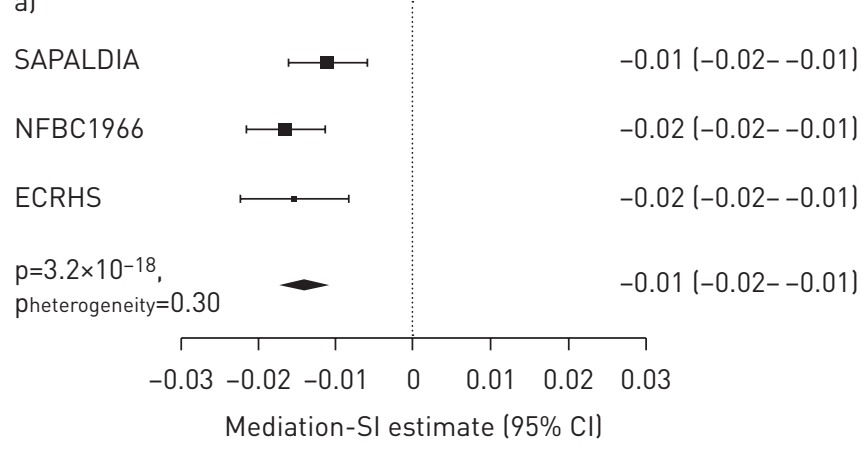

c)

SAPALDIA

NFBC1966

\section{ECRHS}

$p=0.423$,

pheterogeneity $=0.62$

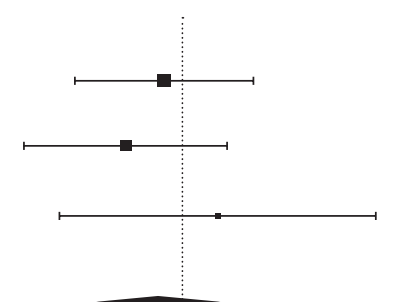

b)

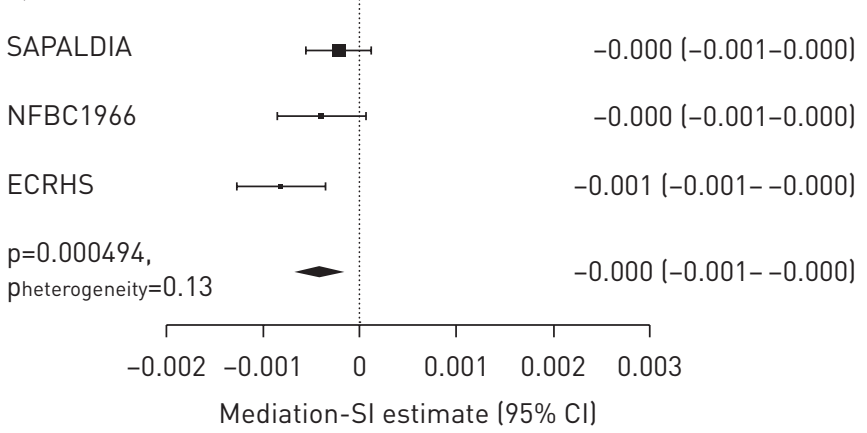

d)

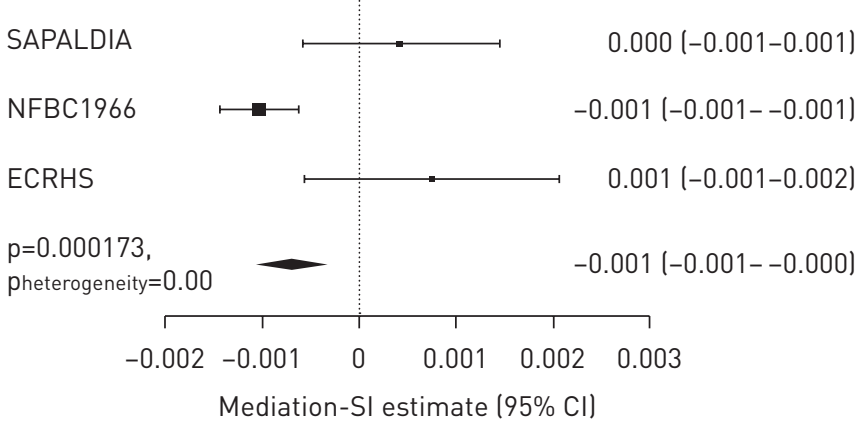

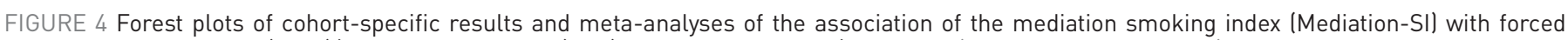

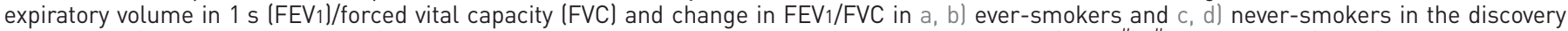
cohorts: a, c) time-point 2 and b, d) prediction. Associations run applying base model adjustment (Mbase ${ }^{\#}$ ). ${ }^{\#}$ : base model (Mbase) epigenome-wide association study was covariate adjusted for age, age squared, height, squared deviation from the mean of height, sex and interaction terms of

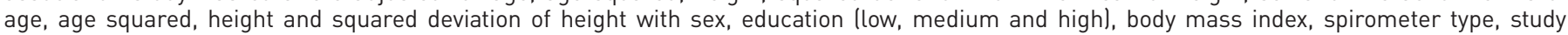
centre, and cell composition. Prediction models were additionally adjusted for FEV1/FVC at time-point 1. 

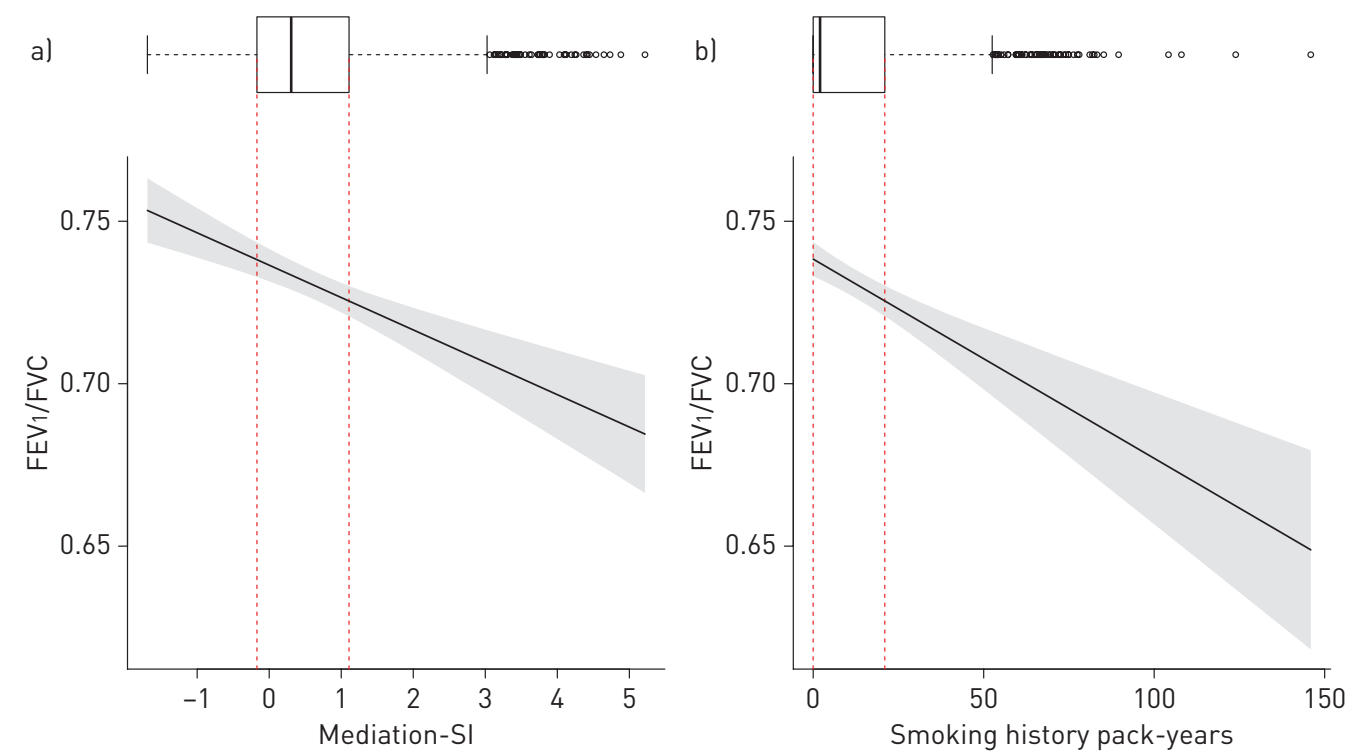

FIGURE 5 Distribution and association ${ }^{\#}$ of a) mediation smoking index (Mediation-SI) ${ }^{\text {I }}$ and b) self-reported smoking history (pack-years) with forced expiratory volume in $1 \mathrm{~s}$ (FEV1)/forced vital capacity (FVC) with $95 \%$ confidence intervals (shaded). Box plots of a) Mediation-SI (median (range) $0.3(-1.7-5.2)$ ) and b) pack-years (median (range) 2.0 (0-145.9)) in all participants of SAPALDIA are shown at the top of each panel. Red dotted lines indicate box plot interquartile range (IQR) borders. Whiskers indicate 1.5 IQR of the lower and upper quartile; outliers are indicated. For analogous figures for associations of Mediation-SI with FEV1 and FVC, see supplementary figures S6 and S7, respectively. " : associations were adjusted for the base model (Mbase): age, age squared, height, squared deviation from the mean of height, sex and interaction terms of age, age squared, height and squared deviation of height with sex, education (low, medium and high), body mass index, spirometer type, study centre, and cell composition. ": Mediation-SI can be constructed for all participants irrespective of their smoking status. The Mbase-adjusted model explained $17.5 \%$ of the variance in the outcome. The Mbase-adjusted model additionally adjusted for the Mediation-SI explained $19.6 \%$ of the FEV $1 /$ FVC variance (total adjusted $\mathrm{R}^{2}=0.196$ ) of which $2.8 \%$ of the variance was specifically explained by the Mediation-SI variable. This was comparable to the variance explained by the Mbase-adjusted model additionally adjusted for pack-years and smoking status corresponding to the Msmok model $\left(R^{2}=0.198\right.$, and with $1.6 \%$ of the variance specifically explained by the pack-years variable). Model including both smoking adjustments (Msmok and additionally Mediation-SI) explained $20.1 \%$ of the $\mathrm{FEV}_{1} / \mathrm{FVC}$ variance.

The assessment of a second DNAme smoking score (Lung-Function-Genes-SI), based on smoking-related CpGs located in 18 GWAS-identified lung function candidate genes (supplementary table S24), showed less prominent associations with lung function (strongest association observed in ever-smokers for FEV1: $\beta \pm$ SE $-0.196 \pm 0.053 ; p=0.0002$ ) (supplementary table S25).

\section{Discussion}

The understanding of how environmental exposure and disease are related to site-specific DNAme status is growing $[18,19]$. Our agnostic EWAS on lung function contributes to this body of evidence. Lung function-associated DNAme markers were strongly enriched for smoking-associated loci. More than 50 known smoking CpGs were consistently, and in several cases causally, associated with lung function and its change in adults. The current agnostic approach converges with recent results of DNAme-lung function studies $[4,6,7]$ that were a priori focusing on smoking-related loci, and included pyrosequencing in blood [7] and lung tissue [4] of some of our strongest association signals, including AHRR hypomethylation at cg05575921 and cg21161138, cg05951221 and cg21566642 (ALPPL2), and cg06126421 (IER3). A methylation index integrating 10 DNAme that reportedly mediate the effect of smoking on lung function [4] was associated with lung function level and its change in adults.

Smoking is an important risk factor for poor lung function and accelerated decline. Several EWASs identified a large number of differentially methylated CpG markers to be associated with smoking [2-4]. In particular, the hypomethylation of cg05575921, a CpG located in the third intron of the aryl hydrocarbon receptor repressor $(A H R R)$ gene, investigated for lung function and respiratory symptoms [4], stands out as a robust indicator of smoking status and smoking history [20]. Given the consistency of the associations observed for cg05575921 and the smoking index containing it in this study, the latter may have potential as a biomarker of clinical utility in predicting smoking-related morbidity and mortality [20, 21]. The positive direction of effects observed in identified DNAme-lung function association is in accordance with the 

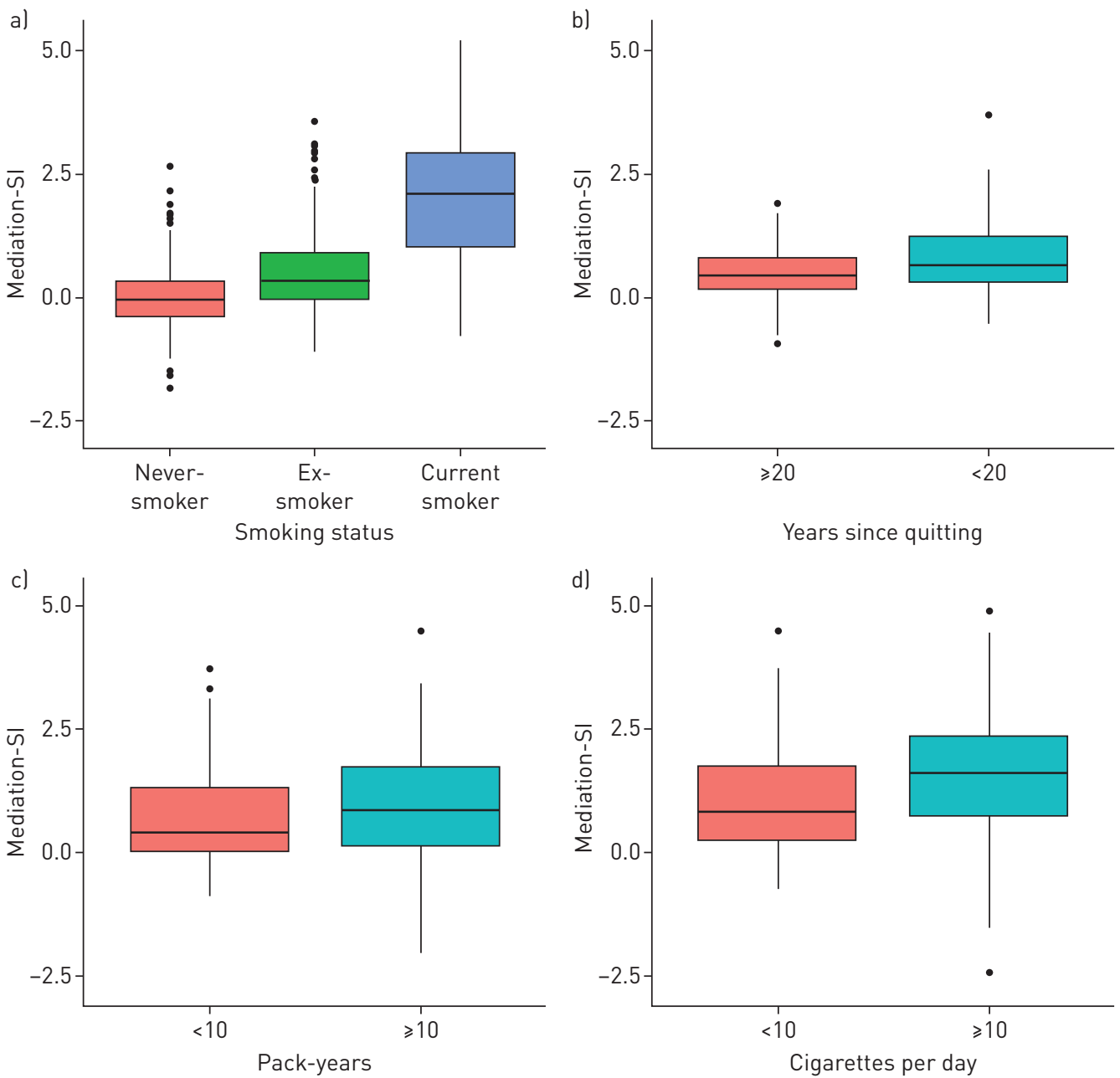

FIGURE 6 Distribution of adjusted mediation smoking index (Mediation-SI) in SAPALDIA at time-point 2. a Smoking status: adjusted for age, sex and education. Never-smokers $(n=395)$, ex-smokers $(n=356)$ and current smokers ( $n=211)$. b) Years since quitting: adjusted for age, sex, education, pack-years and cigarettes per day. Ex-smokers ( $n=356)$. c) Pack-years: adjusted for age, sex, education and cigarettes per day. Current smokers (n=211). d) Cigarettes per day: adjusted for age, sex, education and pack-years. Current smokers $(\mathrm{n}=211)$. Data are presented as median with interquartile range (IQR) (boxes) and 1.5 IQR of the lower and upper quartile (whiskers); outliers are indicated.

reported hypomethylation of smoking-related DNAme sites. The identified lung function-associated CpGs in this study have been previously reported to be associated with smoking-related molecular phenotypes [22], with increased risk of noncommunicable disease, including cancer [20, 23], and with epigenetically defined accelerated ageing [24].

Whether most smoking-related DNAme markers are only markers of exposure or indirectly associated with lung function [7] or whether some inform on causal disease pathways cannot be answered conclusively by the current study. First, DNAme may just be a more precise measure of smoking exposure than self-reporting, as AHRR DNAme was previously shown to correlate with genetic smoking dependency [20]. Second, DNAme identified by previous smoking EWASs [2, 4] may not exclusively have picked up methylation effects of smoking, but methylation related to phenotypes also affected by smoking. In this case, the observed DNAme-lung function associations may result from comorbidity between lung function and other smoking-related phenotypes. However, some of the results are consistent with a causal disease pathway. First, Mendelian randomisation results support causal effects from some DNAme. Unfortunately, no genetic instrument was available for the top ranked $A H R R$ signal. Second, our report confirms nine CpGs, including cg05575921 (AHRR), previously shown to mediate the effect of smoking of lung function [4]. The observation that many smoking DNAme-lung function associations withstood smoking adjustment is consistent with the mediating role of DNAme between smoking behaviour (more distant predictor) and lung function. Third, smoking was also observed to influence methylation in lung tissue at several lung function CpGs, including at $\operatorname{cg} 05575921$ in $A H R R$, and these methylation levels correlated with $A H R R$ 
gene expression [25] and expression of other genes [4]. Hypotheses for a mediating and causal role of smoking-related DNAme include altered AHRR DNAme inducing altered phase 2 enzyme activity and toxicant metabolism, and altered inflammatory pathways in the lung [7]. Other inhalants impacting on the same pathways could in part explain the observed enrichment for smoking DNAme among never-smokers. Methylation of $A H R R \operatorname{cg} 05575921$ was previously associated with lung function and chronic bronchitis in never-smokers [7]. Maternal smoking, passive smoking and environmental exposures other than cigarette smoking (e.g. air pollution) are known to modify DNAme patterns across the genome [26-32]. Maternal smoking during pregnancy has been shown to alter the offspring's DNA markers in a number of genes known to contain smoking-related CpGs [27, 28] and some of these epigenetic patterns, including in $A H R R$, persist to adulthood [29].

From our findings in two well-characterised childhood birth cohorts, there was no evidence for shared common epigenetic mechanisms underlying lung function in adults and children. The comparison was driven by results from the lung function EWAS in adults, given sample size limitations in the available birth cohorts. Lung function in childhood versus adulthood is expected to be influenced in part by different biological processes. The nonreplication of the mostly smoking-related lung function DNAme signals might reflect the nonsmoking status of the children and adolescents. Our findings in SAPALDIA point to a dose-response effect of smoking history and intensity on the smoking index. Effects of maternal exposure in utero, passive smoking or other inhalants on smoking DNAme are likely smaller than the effects of active smoking [30]. Our EWAS findings generally showed an age-related increase in number and strength of DNAme-lung function associations in adults, despite covariate adjustment for age, as also observed by others [6]. This result is consistent with the observed dose-response effect of smoking and possibly other inhalants on DNAme. However, the inherent interdependency of lung function decline, cumulative smoking exposure and DNAme with ageing prohibits attributing associations to single factors.

A systematic review of peripheral DNAme associated with lung function in population-based cohorts pointed to the lack of consistent evidence [5]. Epigenome-wide DNAme profiling studies of lung tissue suggested DNAme in genes such as NOS1AP, TNFAIP2 and CHRM1 to be associated with COPD [13, 14]. An EWAS meta-analysis, adjusted for smoking status and pack-years, identified differential DNAme related to COPD and lung function in Koreans. Five loci (CTU2, USP36, ZNF516, KLK10 and CPT1B) were associated with at least two respiratory traits [12]. Evidence of associations in the current EWAS was only observed for 12 out of 376 CpGs associated with lung function phenotypes in these previous studies. This inconsistency may be due to differences in population ancestry, disease status, exposure status, tissue-specific methylation or covariate adjustment. Furthermore, limited sample size and false discovery findings could contribute to nonreplication, as could the absence of post-bronchodilation lung function in the current EWAS. However, our results confirm the associations of two recently published populationbased reports $[4,6]$ investigating smoking, DNAme and lung function. Both reports and our results reveal the same smoking CpGs as prominent signals.

The strength of this EWAS investigation is the robust and extensive study design with availability of repeat measures of DNAme and spirometry data in the same cohort participants, as well as its population-based design. The utilisation of a multilevel analysis scheme, including cross-sectional and longitudinal EWAS analyses at two time-points in the same participants, and EWAS with and without smoking adjustment in all participants and in never-smokers, allowed for a better understanding of lung function DNAme being affected by ageing and smoking. The lung function-associated smoking index derived is building on robust evidence that DNAme in blood is correlated with DNAme and gene expression in lung tissue [4, 23, 33], and that it is a valid biomarker for capturing the effect of smoking on DNAme in the lung [7, 20].

There are several limitations to this study. Limitations in sample size may explain the inability to find association signals in never-smokers and therefore signals common to lung function in childhood and adulthood. The estimation of decline in lung function from only two spirometry time-points is likely to misclassify decline. Additionally, not all replication cohorts had data available for more than one time-point. Pre-bronchodilation lung function is less robust than post-bronchodilator values and may increase variability of the findings. The meta-analysed EWAS results of the cross-sectional analyses showed evidence of inflation (inflation factor $\lambda>1.1$ ) indicating insufficient genomic control; however, adjusting for genomic inflation did not alter our main results. The relevance of the smoking index derived from CpGs in or close to lung function GWAS genes can be questioned given evidence on the complex trans-regulation of gene expression [34].

In conclusion, our agnostic investigation shows that DNAme at CpGs related to smoking behaviour are the predominant signals associated cross-sectionally and prospectively with lung function in adults. The findings stimulate further research into the involvement of smoking-related CpGs in lung functionrelevant mechanisms and potentially their role as exposure markers beyond active smoking. From our 
EWAS results it has become clear that larger samples are required to confidently identify CpGs involved in lung function and its age-related decline in persons who never smoked.

Acknowledgements: For a full list of acknowledgements for each cohort, please refer to the supplementary material. We gratefully acknowledge the contribution of co-author John M. Starr, who died prior to the publication of this manuscript.

Conflict of interest: M. Imboden has nothing to disclose. M. Wielscher has nothing to disclose. F.I. Rezwan has nothing to disclose. A.F.S. Amaral has nothing to disclose. E. Schaffner has nothing to disclose. A. Jeong has nothing to disclose. A. Beckmeyer-Borowko has nothing to disclose. S.E. Harris reports grants from Medical Research Council, Biotechnology and Biological Sciences Research Council, Age UK and The Wellcome Trust, during the conduct of the study. J.M. Starr has nothing to disclose. I.J. Deary reports grants from Age UK and Medical Research Council, during the conduct of the study. C. Flexeder has nothing to disclose. M. Waldenberger has nothing to disclose. A. Peters has nothing to disclose. H. Schulz reports grants from German Federal Ministry of Education and Research (BMBF), during the conduct of the study. S. Chen has nothing to disclose. S.K. Sunny has nothing to disclose. W.J.J. Karmaus has nothing to disclose. Y. Jiang has nothing to disclose. G. Erhart has nothing to disclose. F. Kronenberg has nothing to disclose. R. Arathimos has nothing to disclose. G.C. Sharp has nothing to disclose. A.J. Henderson reports grants from Medical Research Council and Wellcome Trust, during the conduct of the study. Y. Fu has nothing to disclose. P. Piirilä has nothing to disclose. K.H. Pietiläinen has nothing to disclose. M. Ollikainen has nothing to disclose. A. Johansson has nothing to disclose. U. Gyllensten has nothing to disclose. M de Vries has nothing to disclose. D.A. van der Plaat has nothing to disclose. K. de Jong has nothing to disclose. H.M. Boezen has nothing to disclose. I.P. Hall reports grants from GSK and Boehringer Ingelheim, outside the submitted work. M.D. Tobin reports grants from Pfizer and GSK, outside the submitted work. M-R. Jarvelin has nothing to disclose. J.W. Holloway reports grants from European Union and National Institutes of Health, during the conduct of the study. D. Jarvis reports grants from European Union, Medical Research Council and Asthma UK, during the conduct of the study. N.M. Probst-Hensch has nothing to disclose.

Support statement: This work has been conducted within the Aging Lungs in European Cohorts (ALEC) project, funded from the European Union's Horizon 2020 research and innovation programme under grant agreement 633212. The funding agency had no role in the design, data collection and analysis of the data. Cohort-specific funding details are provided in the supplementary material. Funding information for this article has been deposited with the Crossref Funder Registry.

\section{References}

1 Klimentidis YC, Vazquez AI, de Los Campos G, et al. Heritability of pulmonary function estimated from pedigree and whole-genome markers. Front Genet 2013; 4: 174.

2 Joehanes R, Just AC, Marioni RE, et al. Epigenetic signatures of cigarette smoking. Circ Cardiovasc Genet 2016; 9: 436-447.

3 Gao X, Jia M, Zhang Y, et al. DNA methylation changes of whole blood cells in response to active smoking exposure in adults: a systematic review of DNA methylation studies. Clin Epigenetics 2015; 7: 113.

4 de Vries M, van der Plaat DA, Nedeljkovic I, et al. From blood to lung tissue: effect of cigarette smoke on DNA methylation and lung function. Respir Res 2018; 19: 212.

5 Machin M, Amaral AF, Wielscher M, et al. Systematic review of lung function and COPD with peripheral blood DNA methylation in population based studies. BMC Pulm Med 2017; 17: 54.

6 Carmona JJ, Barfield RT, Panni T, et al. Metastable DNA methylation sites associated with longitudinal lung function decline and aging in humans: an epigenome-wide study in the NAS and KORA cohorts. Epigenetics 2018; 13: 1039-1055.

7 Kodal JB, Kobylecki CJ, Vedel-Krogh S, et al. AHRR hypomethylation, lung function, lung function decline and respiratory symptoms. Eur Respir J 2018; 51: 1701512.

8 Houseman EA, Accomando WP, Koestler DC, et al. DNA methylation arrays as surrogate measures of cell mixture distribution. BMC Bioinformatics 2012; 13: 86.

9 Aryee MJ, Jaffe AE, Corrada-Bravo H, et al. Minfi: a flexible and comprehensive Bioconductor package for the analysis of Infinium DNA methylation microarrays. Bioinformatics 2014; 30: 1363-1369.

10 Willer CJ, Li Y, Abecasis GR. METAL: fast and efficient meta-analysis of genomewide association scans. Bioinformatics 2010; 26: 2190-2191.

11 Quanjer PH, Stanojevic S, Cole TJ, et al. Multi-ethnic reference values for spirometry for the 3-95-yr age range the global lung function 2012 equations. Eur Respir J 2012; 40: 1324-1343.

12 Lee MK, Hong Y, Kim SY, et al. Epigenome-wide association study of chronic obstructive pulmonary disease and lung function in Koreans. Epigenomics 2017; 9: 971-984.

13 Morrow JD, Cho $\mathrm{MH}$, Hersh $\mathrm{CP}$, et al. DNA methylation profiling in human lung tissue identifies genes associated with COPD. Epigenetics 2016; 11: 730-739.

14 Zinellu A, Sotgiu E, Fois AG, et al. Blood global DNA methylation is decreased in non-severe chronic obstructive pulmonary disease (COPD) patients. Pulm Pharmacol Ther 2017; 46: 11-15.

15 Gaunt TR, Shihab HA, Hemani G, et al. Systematic identification of genetic influences on methylation across the human life course. Genome Biol 2016; 17: 61.

16 Hemani G, Zheng J, Elsworth B, et al. The MR-Base platform supports systematic causal inference across the human phenome. Elife 2018; 7: e34408.

17 Tingley D, Yamamoto T, Hirose K, et al. mediation: R package for causal mediation analysis. J Stat Softw 2014; 59: $1-38$.

18 Stover PJ, James WPT, Krook A, et al. Emerging concepts on the role of epigenetics in the relationships between nutrition and health. J Intern Med 2018; 284: 37-49.

19 Franceschi C, Garagnani P, Morsiani C, et al. The continuum of aging and age-related diseases: common mechanisms but different rates. Front Med 2018; 5: 61. 

morbidity and mortality. Thorax 2017; 72: 646-653.

21 Andersen AM, Philibert RA, Gibbons FX, et al. Accuracy and utility of an epigenetic biomarker for smoking in populations with varying rates of false self-report. Am J Med Genet B Neuropsychiatr Genet 2017; 174: 641-650.

22 Zaghlool SB, Mook-Kanamori DO, Kader S, et al. Deep molecular phenotypes link complex disorders and physiological insult to CpG methylation. Hum Mol Genet 2018; 27: 1106-1121.

23 Teschendorff AE, Yang Z, Wong A, et al. Correlation of smoking-associated DNA methylation changes in buccal cells with DNA methylation changes in epithelial cancer. JAMA Oncol 2015; 1: 476-485.

24 Gao X, Zhang Y, Breitling LP, et al. Relationship of tobacco smoking and smoking-related DNA methylation with epigenetic age acceleration. Oncotarget 2016; 7: 46878-46889.

25 Monick MM, Beach SR, Plume J, et al. Coordinated changes in AHRR methylation in lymphoblasts and pulmonary macrophages from smokers. Am J Med Genet B Neuropsychiatr Genet 2012; 159B: 141-151.

26 Knopik VS, Marceau K, Bidwell LC, et al. Prenatal substance exposure and offspring development: does DNA methylation play a role? Neurotoxicol Teratol 2018; 71: 50-63.

27 Nielsen $\mathrm{CH}$, Larsen A, Nielsen AL. DNA methylation alterations in response to prenatal exposure of maternal cigarette smoking: a persistent epigenetic impact on health from maternal lifestyle? Arch Toxicol 2016; 90: 231-245.

28 Joubert BR, Felix JF, Yousefi P, et al. DNA methylation in newborns and maternal smoking in pregnancy: genome-wide consortium meta-analysis. Am J Hum Genet 2016; 98: 680-696.

29 Richmond RC, Joubert BR. Contrasting the effects of intra-uterine smoking and one-carbon micronutrient exposures on offspring DNA methylation. Epigenomics 2017; 9: 351-367.

30 Reynolds LM, Magid HS, Chi GC, et al. Secondhand tobacco smoke exposure associations with DNA methylation of the aryl hydrocarbon receptor repressor. Nicotine Tob Res 2017; 19: 442-451.

31 de Lichtenfels AJ, van der Plaat DA, de Jong $\mathrm{K}$, et al. Long-term air pollution exposure, genome-wide DNA methylation and lung function in the LifeLines cohort study. Environ Health Perspect 2018; 126: 027004.

32 Hesselbach K, Kim GJ, Flemming S, et al. Disease relevant modifications of the methylome and transcriptome by particulate matter $\left(\mathrm{PM}_{2.5}\right)$ from biomass combustion. Epigenetics $2017 ; 12$ : 779-792.

33 Stueve TR, Li WQ, Shi J, et al. Epigenome-wide analysis of DNA methylation in lung tissue shows concordance with blood studies and identifies tobacco smoke-inducible enhancers. Hum Mol Genet 2017; 26: 3014-3027.

34 Yao C, Joehanes R, Johnson AD, et al. Dynamic role of trans regulation of gene expression in relation to complex traits. Am J Hum Genet 2017; 100: 571-580. 